\title{
PLANAR ARRAY ANTENNA DESIGN ANALYSIS
}

\author{
Bradiey P. Chrisman \\ Air Force Electronic Combat Office \\ Engineering Division \\ Wright-Patterson AFB, Ohio 45433-6503
}

\begin{abstract}
A computer program is presented that can assess the impact of small. random design perturbations on the performance of a planar array antenna of dipoles. Expressions for the expected or average radiation pattem and the change in directivity for a perturbed planar array are developed and used in the program. Translational errors in the positions of the elements, errors in the element's drive amplitude and phase, non-identical element patterns, and missing elements are accounted for in the expected radiation pattem. The user specifies the array's design parameters to include the number of elements in each direction of the array, interelement spacing, aperture distribution; i.e., DolphChebyshev, Taylor, binomial, uniform, or user defined, the dipole's orientation and length, and the tolerance data. The program calculates data points to allow plotting the radiation pattern in the two principal planes of the array, half-power beamwidths, and directivities for specified scan angles and frequency bandwidth for the design and expected arrays. The radiation pattem data can be plotted allowing side lobe comparison between the expected and design arrays. Program results are validated against trends noted in earlier studies on the effects of random errors in arrays.
\end{abstract}




\section{PLANAR ARRAY ANTENNA \\ DESIGN ANALYSIS}

Bradley P. Chrisman

AFECO/EN

WPAFB, OH 45433

\section{INTRODUCTION}

One of the fundamental principles of array antenna theory is that the radiation pattern is determined by the amplitude and phase of the excitation currents over the array, interelement spacing, trequency of operation. and the choice of radiating elements. The antenna designer can, theoretically, specity the form of the currents across the array, the interelement spacings, and elements and expect the resulting radiation pattern to be as predicted. In practice, however. there will be unavoidable errors in the amplitude and phase of currents. the interelement spacing, and the individual element's radiation pattern so that the actual radiation pattem will differ from the theoretical. The agreement between the two depends on how well the desired distribution of the currents across the array, the interelement spacing, and the element's radlation pattern can be achieved.

Errors in an array antenna can be divided into two categories depending on whether they are predictable (systematic) or random (Refs. 21;22;24). Chief among predictable errors is a phenomena known as mutual coupling. The signal emitted from any one of ement will induce a sympathetic excitation in every other element. thus, altering the array's radiation pattem (Rel. 24). Another example is the finite quantization of the phase produced by a digital phase shifter (Ref. 22). The effects of such errors are predictable and the resulting radiation pattem can be computed by classical methods from a knowledge of the array design.
Random errors are caused by the accidental deviation of the array antenna parameters from their design value. An array antenna radiation pattern might differ from the desired pattem because of, (1) errors in the amplitude of the currents at each element. (2) errors in the phase of the current. (3) missing elements (due to catastrophic failure), (4) rotation of the radiating elements, (5) translational errors in the element location, and (6) errors in the radiation pattem of each element (Rel. 22). Although they are small. they are ever-present and can limit the minimum side lobe level that can be achieved just as random noise limits the sensitivity of a radio receiver. In most radar systems, low side lobe levels are very important in minimizing the false target indications and jamming through the side lobes (Ref. 4). Random errors can also cause a reduction in power gain. an error in the direction of the main beam, lower directivity, and deviations in other performance standards.

If errors in an existing antenna can be measured, the pattern can be calculated in the classical manner. However, one cannot predict the exact nature of random errors that might be encountered in some particular antenna from a knowledge of its design. The actual existence of an antenna is required. It is possible, however, to predict in statistical terms the pattern behavior of a collection of antennas. The average value of the pattem and the standard deviation about the average are used to describe the antenna performance. The statistical description of random errors cannot be applied to any particular antenna but applies to the collection of similar antennas whose errors are described by the same statistical parameters (Ref. 22).

Many authors (Refs. 1.2.3.7,8,11,13,14, $16,18,20,21,22,24$ ) have studied the effects of random errors on the radiation pattem of array antennas and developed expressions relating these errors to different performance 
characteristics of the antenna. Random errors considered in earlier studies included amplitude and phase errors at the drive of each element, translational and rotational errors in the position of each element, non-identical element pattems, and the possibility of catastrophic element failures. However. no one author has derived an expression for the average radlation pattern of a planar array with all the aforementioned random errors included.

The purpose of this paper is to derive an expression of the average or expected radiation pattern for a planar array and present a FORTRAN program that incorporates these results to determine the change in an array's side lobe level (radiation pattern), half-power beamwidth, and directivity due to random errors. This effort focuses on the results of three earlier studies conducted by Elliott (Ref. 11), Skolnik (Ref. 22), and Allen (Ref. 1) on the effects of random errors on array performance. Elliott derived an expression for the average radiation pattem for a planar array considering random amplitude and phase errors at the drive of the elements and translational and rotational errors in the positions of the elements. The main conclusion from Elliott's study used in this paper, is that rotational errors were relatively unimportant when compared to the other errors. Skolnik derived an expression for the average radiation pattem for a planar array considering random amplitude and phase errors at the drive of the elements and missing elements (due to catastrophic fallure). Allen extended the study by considering random amplitude and phase errors at the element drive, translational errors in the positions of the elements, non-identical element factors, and missing elements in deriving the average radiation pattern expression for a linear array. Rotational errors in the positions of the elements were not considered in Allen's work based on the previous conclusion from Elliott's work. The expression for the average radiation pattern for a planar array derived in this paper will include all the errors considered by Allen and his formulation and will follow Skolnik's methodology.
Once the average array pattern is computed for a certain scan angles and element excitations, it is a straight forward process to determine the random error effects on the array's side lobe level, half-power beamwidth, and directivity.

A FORTRAN program will be described that computes the change in side lobe level. half-power beamwidth, and directivity based on user input. The user specifies certain tolerances, excitation schemes, dimensions of the array, phase tapers, operating wavelength or frequency, and the bandwidth of operation. The program is validated against examples found in the current literature and conclusions noted in earlier studies on the effects of random errors on an planar array's performance standards.

\section{THE EFFECTS OF RANDOM ERRORS ON THE ARRAY PATTERN}

\section{Sources of Errors Affecting Array Performance}

Before we begin the development there are several assumptions that must be considered. First. the excitation coefficients and the positions of the elements actually have some random scatter about their mean or expected values. These expected values may be regarded as averages, taken over a large number of different arrays, or they may be thought of as long-term time averages for a single array whose parameters vary with time in a random fashion (Rets. 11,14,21,22). Second, the root-mean-square errors in the element positions are all small. compared to wavelength, independent random variables which are normally distributed (Gaussian distribution) with zero means and respective variances $\gamma_{x, y z}^{2}$ (Refs. 1,3,11,16.20). Third, the mean-square phase and amplitude errors in the drive current at any element and the mean-square error in the element pattems are all taken to be independent of the errors in any other element. small, and normally distributed. The meansquare phase errors are small compared to pi $(\pi)$ and the mean-square amplitude and 
element pattern errors are small compared to unity (Refs. 1,16,22).

We can write the no-error pattern factor of a planar array, taking into account the element factor, as:

$$
\begin{aligned}
U(\theta, \phi)= & U_{\bullet .} \sum_{m=1}^{M} \sum_{m=1}^{N} a_{m} \\
& x \exp \left[j k \left[(m-1)\left(v-v_{0}\right)\right.\right. \\
& \left.\left.+(n-1)\left(U-u_{0}\right)\right]\right]
\end{aligned}
$$

where:

$$
\begin{aligned}
& v=k d_{x} \sin \theta \cos \phi, \\
& v_{0}=k d_{x} \sin \theta_{0} \cos \phi_{0}, \\
& u=k d_{y} \sin \theta \sin \phi, \\
& u_{0}=k d_{y} \sin \theta_{0} \sin \phi_{0},
\end{aligned}
$$

$d_{x}$ and $d_{y}$ are the interelement spacings in the $x$ and $y$ directions, respectively, $k=2 \pi / \lambda$, and $\lambda$ is the wavelength. The planar array geometry is shown in Figure 1. Further, we are assuming the array is linearty phased to point the main beam in the direction $\theta_{0}, \phi_{0}$ as shown in Figure 2. The function $U_{\theta}(\theta, \phi)$ is the unnormalized element factor which is the average pattern of the array elements in the array environment.

Instead of actually realizing the field in equation (1), one would in practice obtain a field:

$$
\begin{aligned}
U(\theta, \phi)= & \sum_{m=1}^{M} \sum_{m=1}^{N} U_{0.0 ; m}(\theta, \phi) \\
& X a_{m m}^{\cdot} \exp \left[-j(m-1) v_{0}\right] \\
& X \exp \left[-j(n-1) u_{0}\right] \\
& X \exp \left(\delta_{m m}\right) \exp \left(j k r_{m}^{\prime} \cdot \hat{r}\right)
\end{aligned}
$$

where $U_{\theta .0 ; m m}(\theta, \phi)$ is the actual pattern of the $m^{\text {th }}$ element in its array environment. with respect to a coordinate system centered on the $\mathrm{mn}^{\text {th }}$ element. The quantity $a_{m}$ represents the value of a with error and $\delta$ is the phase error of the $\mathrm{mn}^{\text {th }}$ element drive. The exponential expressing the phase delay of the elements at the far-field point is generalized to allow for mechanical error in antenna placement. The vector dot product is written in spherical coordinates as:

$$
\begin{aligned}
r_{m m}^{\prime} \bullet \hat{r}= & x_{m m} \sin \theta \cos \phi \\
& +y_{m} \sin \theta \sin \phi+z_{m m} \cos \theta
\end{aligned}
$$

where the "center of radiation" of the $\mathrm{mn}^{\text {th }}$ element is at $x_{m n}, y_{m}, z_{m n}$ (Ref. 1).

\section{Reduction of Errors to Pure Amplitude and Phase Errors}

As we can see from equation (6), many errors manifest themselves as amplitude and phase errors. This is easily seen by recasting equation (6) in the form:

$$
\begin{aligned}
U(\theta, \phi)= & U_{\theta . \phi}(\theta, \phi) \sum_{m=1}^{M} \sum_{n=1}^{N} b_{m n}(\theta, \phi) \\
& X \exp \left[j \Psi_{m m}(\theta, \phi)\right] \\
& X \exp \left[j k \left[(m-1)\left(v-v_{0}\right)\right.\right. \\
& \left.\left.+(n-1)\left(u-u_{0}\right)\right]\right]
\end{aligned}
$$

where for particular angles $\theta$ and $\phi$, the apparent amplitude of the $\mathrm{mn}^{\text {th }}$ element is given by:

$$
b_{m m}(\theta, \phi)=\left[U_{0,0, m n}(\theta, \phi) / U_{\theta, \theta}(\theta, \phi)\right] a_{m n}^{\prime}
$$

and the apparent phase error term, $\Psi_{m m}(\theta, \phi)$, by:

$$
\begin{aligned}
\Psi_{m m}(\theta, \phi)= & \delta_{m m}+k\left[x_{m m}-(m-1) d_{x}\right] \sin \theta \cos \phi \\
& +k\left[y_{m m}-(n-1) d_{y}\right] \sin \theta \sin \phi \\
& +k z_{m m} \cos \theta
\end{aligned}
$$


Statistics of the Apparent Amplitude Error.

We can write the element factor of the $\mathrm{mn}^{\mathrm{th}}$ element for particular angles $\theta$ and $\phi$ as:

$$
U_{\theta, 0, m m}(\theta, \phi)=U_{\theta, 0}(\theta, \phi)\left[1+\mu_{m m}(\theta, \phi)\right]
$$

where the $\mu_{\mathrm{mm}}(\theta . \phi)$ are samples of a random variable and are assumed to be normally distributed with a zero mean and variance $\sigma_{\mu}^{2}(\theta, \phi)$.

The amplitude error of the drive to the $\mathrm{mn}^{\text {th }}$ element is handled in a similar manner by assigning a random component, $\Delta_{m m}$, to the drive amplitude. This is assigned in a multiplicative manner as (Ref. 1):

$$
a_{m n}^{\prime}=\left(1+\Delta_{m n}\right) a_{m n}
$$

where the $\Delta_{m n}$ are samples of a random variable and are assumed to be normally distributed with a zero mean and variance $\sigma_{\Delta}^{2}$.

write:

$$
\text { Adding a reliability factor, } r_{m n} \text {, we can }
$$

$$
a_{m n}^{\prime}=r_{m n}\left(1+\Delta_{m n}\right) a_{m n}
$$

where $r_{m}=1$ with a probability $P$. and zero with probability $1-P$. Finally, we can write (Ref. 1):

$$
b_{m n}=\left[1+\mu_{m n}(\theta, \phi)\right]\left[1+\Delta_{m n}\right] r_{m n} a_{m m}
$$

With these assumptions. we can write the mean and mean-square of the $\mathrm{mn}^{\text {th }}$ element's apparent amplitude as:

$$
\overline{b_{m n}}(\theta, \phi)=a_{m m} P
$$

and

$$
\begin{aligned}
\overline{\mathrm{b}_{m n}^{2}}(\theta, \phi)= & \operatorname{Pa}_{m n}^{2}\left(1+\sigma_{\mu}^{2}(\theta, \phi)\right. \\
& \left.+\sigma_{\Delta}^{2}+\sigma_{\mu}^{2}(\theta, \phi) \sigma_{\Delta}^{2}\right)
\end{aligned}
$$

Separating the catastrophic failure from the $\Delta_{m}$ factor. the variances in the $\mu$ and $\Delta$ cross product term will be much less than unity in practice and their product can be ignored in equation (16) without much loss of accuracy (Ref. 1). Letting:

$$
\sigma_{A}^{2}(\theta, \phi) \approx \sigma_{\mu}^{2}(\theta, \phi)+\sigma_{\Delta}^{2}
$$

we can write equation (16) as:

$$
\overline{b_{m n}^{2}}(\theta, \phi)=P a_{m n}^{2}\left[1+\sigma_{A}^{2}(\theta, \phi)\right]
$$

where $\sigma_{A}^{2}(\theta, \phi)$ is the amplitude variance.

\section{Statistics of the Apparent Phase Error.}

In finding the expected power pattern we will be taking the complex conjugate of equation (10), multiplying the result by equation (10), and then finding the average denoted by an overbar, such that:

$$
\begin{aligned}
& \overline{\exp \left[j\left[\Psi_{m m}(\theta, \phi)-\Psi_{p q}(\theta, \phi)\right]\right]} \\
&=\frac{\operatorname{exp[j(\delta _{mm}-\delta _{pq})]}}{} \\
& \times \overline{\exp \left[j k\left(x_{m n}-x_{p q}\right) \sin \theta \cos \phi\right]} \\
& \times \overline{\exp \left[j k\left(y_{m m}-y_{p q}\right) \sin \theta \sin \phi\right]} \\
& \overline{\exp \left[j k\left(z_{m m}-z_{p q}\right) \cos \theta\right]}
\end{aligned}
$$

where it is assumed that $\delta_{m n}, \delta_{p q}, x_{m n}, x_{p q}, y_{m n}, y_{p q}$, $z_{m,}$ and $z_{p q}$ are all statistically independent random variables (Ref. 22).

Assuming that the phase error $\delta$ (we have dropped the subscripts for simplicity) is described by the gaussian probability density function with zero mean (Ref. 22):

$$
P_{1}(\delta)=\left[1 /\left(2 \pi \overline{\delta^{2}}\right)^{4}\right] \exp \left[-\delta^{2} /\left(2 \overline{\delta^{2}}\right)\right]
$$

where $\bar{\delta}^{2}$ is the variance (generally denoted $\sigma^{2}$ ) or mean-square value of the deviation about the average, which average here is zero. The probability density function $p_{1}(\delta)$, when multiplied by the intinitesimal $d \delta$, gives the probability of finding the variable $\delta$ between the values of $\delta$ and $\delta+d \delta$. The gaussian probability density function is chosen because it is a good 
description of many types of errors that occur in practice (Ref. 22). The gaussian assumption will be asymptotically true if the phase error $\delta$ is due to a number of causes and such errors are small so that a linear relation exists between the cause of the phase error and the error itself (Ref. 22). By asymptotic. it is meant that as the sample size is increased, the dispersion in the associated sampling distribution of the mean decreases, reflecting the increased clustering of the sample means such that the gaussian distribution is obtained (Ref 18).

Let $y=\delta_{m}-\delta_{p q}$ so that the problem becomes that of finding the average value of $\theta^{y}$. From the definition of the average value we have (Ref 22):

$$
\overline{\exp (j y)}=\int_{-\infty}^{\infty} \exp (j y) p(y) d y
$$

where $p(y)$ is the probability density function for the variable $y$. The probability density function for $\delta$ is given by equation (20) and is needed in determining $p(y)$. The variable $y$ is a function of two independent variables $\delta_{m}$ and $\delta_{\text {g }}$ (we have defined them to be independent so long as $(m \neq p, n \neq q)$. The joint probability density function for the two independent variables is the product of the individual variable's densities (Ref. 22):

$$
p\left(\delta_{m n}, \delta_{p q}\right)=p_{1}\left(\delta_{m n}\right) p_{1}\left(\delta_{p q}\right)=p_{1}\left(y+\delta_{p q}\right) p_{1}\left(\delta_{p q}\right)
$$

By substituting $p_{1}(\delta)$ given in equation (20) into the integral for $p(y)$, we find the average value of $\theta^{\text {ty }}$ to be (Ref. 22):

$\overline{\exp (j y)}=\int_{-\infty}^{\infty} \cos (y) p(y) d y+j \int_{-\infty}^{\infty} \sin (y) p(y) d y$

The second term is zero, provided the integral exists, since an odd function integrated from $-\infty$ to $+\infty$ is zero $(\sin (y)$ is an odd function, $p(y)$ is an even function). Substituting the PDF of equation (20) into equation (23) and using (Ref. 9):

$$
\begin{aligned}
\int_{0}^{\infty} \exp \left(-a^{2} x^{2}\right) \cos (2 p x) d x & \\
= & {\left[\pi^{4} /(2 a)\right] \exp \left(-p^{2} / a^{2}\right) }
\end{aligned}
$$

to perform the integration on the first integral, we find (Ref. 22):

$$
\overline{\exp (j y)}=\overline{\cos (y)}=\exp \left(-\overline{\delta^{2}}\right)
$$

Following a similar procedure for the second term averaged in equation (19), using (24), we find:

$$
\begin{aligned}
\overline{\exp \left[\overline{j k}\left(x_{m m}-x_{p q}\right) \sin \theta \cos \phi\right]} \\
\left.=\overline{\exp \left(j k \gamma_{x} \sin \theta \cos \phi\right.}\right) \\
=\exp \left[\overline{\gamma_{x}^{2}} k^{2} \sin ^{2}(\theta) \cos ^{2}(\phi)\right]
\end{aligned}
$$

Similarly:

$$
\begin{aligned}
& \overline{\exp \left(\hat{j k} \gamma_{y} \sin \theta \sin \phi\right)} \\
& =\exp \left[\overline{\gamma_{y}^{2}} k^{2} \sin ^{2}(\theta) \sin ^{2}(\phi)\right]
\end{aligned}
$$

and:

$$
\left.\overline{\exp \left(j k \gamma_{2} \cos \theta\right.}\right)=\exp \left[\overline{\gamma_{2}^{2}} k^{2} \cos ^{2}(\theta)\right]
$$

where $\gamma_{y}=y_{m}-y_{p q}$ and $\gamma_{z}=z_{m m}-z_{p q}$.

\section{Power Pattern}

The power, or radiation, pattem is:

$$
\begin{aligned}
|U(\theta, \phi)|^{2} & =U(\theta, \phi) U^{*}(\theta, \phi) \\
= & U_{\theta .0}^{2}(\theta, \phi) \\
& x \sum_{m=1}^{N} \sum_{m=1}^{N} \sum_{p=1}^{M} \sum_{q=1}^{N} b_{m m}(\theta, \phi) b_{p q}^{*}(\theta, \phi) \\
& X \exp \left[\left[\left[\Psi_{m m}(\theta, \phi)-\Psi_{p q}(\theta, \phi)\right]\right]\right. \\
& X \exp \left[j(m-p)\left(v-v_{0}\right)\right] \\
& X \exp \left[(n-q)\left(u-u_{0}\right)\right]
\end{aligned}
$$


Substituting equation (14) for $b(\theta, \phi)$ and $a$ similar expression in terms of $p$ and $q$ for $b_{p q}^{*}(\theta, \phi)$ into $(29)$ we get:

$$
\begin{aligned}
|U(\theta, \phi)|^{2}= & U_{\theta, 0}^{2}(\theta, \phi) \\
& X \sum_{m=1}^{M} \sum_{n=1}^{N} \sum_{p=1}^{M} \sum_{\phi=1}^{N}\left[\left[1+\mu_{m m}(\theta, \phi)\right]\right. \\
& \left.X\left(1+\Delta_{m m}\right) r_{m m} a_{m m}\right] \\
& X\left|\left[1+\mu_{p q}(\theta, \phi)\right]\left(1+\Delta_{p q}\right) r_{p q} a_{p q}^{\cdot}\right| \\
& X \exp \left[j\left[\Psi_{m m}(\theta, \phi)-\Psi_{p q}(\theta, \phi)\right]\right] \\
& X \exp \left[j \left[(m-p)\left(v-v_{0}\right)\right.\right. \\
& \left.\left.+(n-q)\left(u-u_{0}\right)\right]\right]
\end{aligned}
$$

This power pattern is a random quantity since $r, \Delta, \mu$, and $\Psi$ are random variables. Now, we want to obtain the average power pattern. To start. we must separate the summation into two parts by separating those terms in which $m=p, n=q$ from the remaining terms in which $m \neq p, n \neq q$. This is done so we can apply an axiom in probability theory which states that the mean of a product of statistically independent random variables is equal to the product of the means of these random variables. The variables $\Delta_{m}, \Delta_{m}, \mu_{m}(\theta, \phi), \mu_{m q}(\theta, \phi), \Psi_{m}(\theta, \phi)$, $\Psi_{p q}(\theta, \phi)$ are independent if and only if $m \neq p, n$ $\neq q$ (Ref. 22).

Considering only those terms in the power pattern which are statistically independent and applying the aforementioned axiom yields:

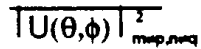

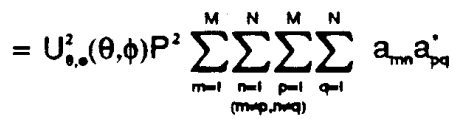

$$
\begin{aligned}
& x \overline{\exp \left[\left(\delta_{m n}-\delta_{p q}\right)+k\left(x_{m n}-x_{p q}\right) \sin \theta \cos \phi\right.} \\
& +\overline{k\left(y_{m m}-y_{p q}\right) \sin \theta \sin \phi} \\
& +\overline{\left.k\left(z_{m m}-z_{p q}\right) \cos \theta\right]} \\
& X \exp \left[j(m-p)\left(v-v_{0}\right)\right] \\
& X \exp \left[j(n-q)\left(u-u_{0}\right)\right]
\end{aligned}
$$

where

$$
\overline{b_{m n}}=P a_{m m}
$$

and

$$
\overline{b_{p q}^{*}}=P a_{p q}^{*}
$$

have been used. In obtaining equation (31) we assumed $\Delta_{m}$ and $\mu_{m}(\theta, \phi)$ are zero mean and that $r_{m m}=P^{m}$ (Ref. 22). Substituting equations (25), (26), (27), and (28) into equation (31), we can write:

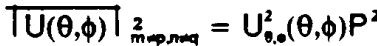

$$
\begin{aligned}
& X \sum_{m=1}^{M} \sum_{\substack{n=1 \\
(m, p=1, n=1}}^{N} \sum_{p=1}^{N} a_{m m} a_{p q}^{*} \\
& X \exp \left(-\overline{\delta^{2}}\right) \\
& x \exp \left(-\overline{\gamma_{x}^{2}} k^{2} \sin ^{2} \theta \cos ^{2} \phi\right) \\
& x \exp \left(-\overline{\gamma_{y}^{2}} k^{2} \sin ^{2} \theta \sin ^{2} \phi\right) \\
& X \exp \left(-\overline{\gamma_{2}^{2}} k^{2} \cos ^{2} \theta\right) \\
& X \exp \left[j(m-p)\left(v-v_{0}\right)\right] \\
& X \exp \left[j(n-q)\left(u-u_{0}\right)\right]
\end{aligned}
$$


Now for the terms $m=p$ and $n=q$, we find:

$$
\overline{\mathrm{U}(\theta, \phi)}]_{m \rightarrow-\Omega}^{2}=\mathrm{U}_{\theta .0}^{2}(\theta, \phi) \sum_{m=1}^{M} \sum_{m=1}^{N} \overline{\mathrm{b}_{m n}^{2}(\theta, \phi)}
$$

where $\overline{b_{m n}^{2}(\theta, \phi)}$ is given by equation (18). We can write equation (34) as:

$$
\begin{aligned}
\widehat{T U(\theta, \phi)}]_{m-\rho, m}^{2}= & U_{0.0}^{2}(\theta, \phi) P\left[1+\sigma_{A}^{2}(\theta . \phi)\right] \\
& \times \sum_{m=1}^{M} \sum_{n=1}^{N} a_{m m}^{2}
\end{aligned}
$$

The average power pattern is the sum of the $m \neq p, n \neq q$ and $m=p, n=q$ terms (Ret. 22):

$$
\begin{aligned}
& \overline{U(\theta, \phi)}^{2}=U_{0.0}^{2}(\theta, \phi) P^{2} \exp \left(-\bar{\delta}^{2}\right) \\
& X \exp \left[-k^{2}\left(\bar{\gamma}_{x}^{2} \sin ^{2} \theta \cos ^{2} \phi\right.\right. \\
& \left.\left.+\overline{\gamma_{y}^{2}} \sin ^{2} \theta \sin ^{2} \phi+\overline{\gamma_{2}^{2}} \cos ^{2} \theta\right)\right]
\end{aligned}
$$

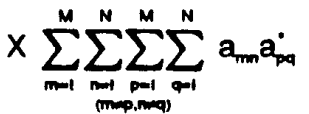

$$
\begin{aligned}
& X \exp \left[j I(m-p)\left(v-v_{0}\right)\right. \\
& \left.\left.+(n-q)\left(u-u_{0}\right)\right]\right] \\
& +U_{\theta, \phi}^{2}(\theta, \phi)\left[1+\sigma_{A}^{2}(\theta, \phi)\right] \\
& X \sum_{m=1}^{M} \sum_{m=1}^{N} a_{m}^{2}
\end{aligned}
$$

In order to simplify matters, we will let:

$$
\begin{aligned}
\Psi_{A P}(\theta, \phi)= & \exp \left[-\left[\overline{\delta^{2}}+k^{2}\left(\overline{\gamma_{x}^{2}} \sin ^{2} \theta \cos ^{2} \phi\right.\right.\right. \\
& \left.\left.\left.+\overline{\gamma_{y}^{2}} \sin ^{2} \theta \sin ^{2} \phi+\overline{\gamma_{z}^{2}} \cos ^{2} \theta\right)\right]\right]
\end{aligned}
$$

Adding the terms $m=p$ and $n=q$ to the first summation and subtracting them as separate terms, so as not to include them twice. the average power pattern becomes (Ref. 22):

$$
\begin{aligned}
\left.\overline{U(\theta, \phi)}\right|^{2}= & P^{2} \Psi_{A P}(\theta, \phi) U_{\theta, \theta}^{2}(\theta, \phi) \\
& X \sum_{m=1}^{M} \sum_{n=1}^{N} \sum_{p=1}^{M} \sum_{\phi=1}^{N} a_{m m} a_{\phi \phi}^{\cdot} \\
& X \exp \left[j \left[(m-p)\left(v-v_{0}\right)\right.\right. \\
& \left.\left.+(n-q)\left(u-u_{0}\right)\right]\right] \\
+ & U_{0.0}^{2}(\theta, \phi)\left[\left[1+\sigma_{A}^{2}(\theta, \phi)\right] P\right. \\
& \left.-P^{2} \Psi_{A P}(\theta, \phi)\right] \\
& \times \sum_{m=1}^{M} \sum_{n=1}^{N} a_{m n}^{2}
\end{aligned}
$$

Simplifying, we find:

$$
\begin{aligned}
\widehat{\overline{U(\theta, \phi)}]^{2}=} & P^{2} \Psi_{A P}(\theta, \phi)\left|U_{0}(\theta, \phi)\right|^{2} \\
& +U_{0.0}^{2}(\theta, \phi) \\
& X\left[\left[1+\sigma_{A}^{2}(\theta, \phi)\right] P-P^{2} \Psi_{A P}(\theta, \phi)\right] \\
& \times \sum_{m=1}^{M} \sum_{m=1}^{N} a_{m}^{2}
\end{aligned}
$$

Equation (39) is the sought for result of our analysis. This equation has the same form as Allen's equation (82) for a linear array (Ref. 1). The effect of random errors is to produce an average power pattern that is the superposition of two terms. The first term is the no-error power pattern multiplied by the fraction of the elements remaining and the apparent phase error. The second term depends on the apparent amplitude and phase errors and the fraction of elements remaining and is directional only by the element factor and the terms in the apparent phase error. 


\section{Random Error Effects on Array Directivity}

In order to predict the effect of random errors on the array's directivity, it is useful to formulate the average power pattern of the array normalized to a no-error peak value of unity (Ref. 1). Then, by definition, the average power pattern is:

$$
\overline{P(\theta, \phi)}=\overline{U(\theta, \phi) U^{*}(\theta, \phi)}=\left.\overline{\mid U(\theta, \phi)}\right|^{2}
$$

where $\overline{U(\theta, \phi)}{ }^{2}$ is given by equation (39). In preparation for what follows, in equation (39) let:

$$
A(\theta, \phi)=1+\sigma_{A}^{2}(\theta, \phi)-P \Psi_{A P}(\theta, \phi)
$$

so that equation (39) becomes:

$$
\begin{aligned}
\overline{\mathrm{U}(\theta, \phi)}^{2}= & \mathrm{P}^{2} \Psi_{A P}(\theta, \phi)\left|\mathrm{U}_{0}(\theta, \phi)\right|^{2} \\
& +\mathrm{U}_{0, \phi}^{2}(\theta, \phi) P A(\theta, \phi) \sum_{m=1}^{M} \sum_{m=1}^{N} a_{m m}^{2}
\end{aligned}
$$

Now, for the directivity calculation the absolute value of $\left.\overline{\mathrm{U}(\theta, \phi)}\right|^{2}$ is immaterial, so we can divide equation (42) by $P^{2} \Psi_{A P}(\theta, \phi)$ and use the form (Ref. 1):

$$
\begin{aligned}
\left.\overline{\mathrm{U}(\theta, \phi)}\right|^{2} & =\left|\mathrm{U}_{0}(\theta, \phi)\right|^{2} \\
& +\left[U_{0.0}^{2}(\theta, \phi) / P\right] \varepsilon^{2}(\theta, \phi) \sum_{m=1}^{M} \sum_{n=1}^{N} a_{m}^{2}
\end{aligned}
$$

where:

$$
\varepsilon^{2}(\theta, \phi)=A(\theta, \phi) / \Psi_{A P}(\theta, \phi)
$$

If we define a no-error efficiency ( $\eta$ ) of the array amplitude taper as (Ref. 1):

$$
\eta=\left[\sum_{m=1}^{M} \sum_{n=1}^{N} a_{m n}\right]^{2 /}\left[M N \sum_{m=1}^{M} \sum_{n=1}^{N} a_{m m}^{2}\right]
$$

we will obtain (Ref. 1):

$$
\sum_{m=1}^{M} \sum_{n=1}^{N} a_{m}^{2}=\left[\sum_{m=1}^{M} \sum_{n=1}^{N} a_{m}\right]^{2} /(n M N)
$$

and:

$$
\sum_{m=1}^{M} \sum_{m=1}^{N} a_{m}^{2}=\left|U_{0}\left(\theta_{0}, \phi_{0}\right)\right|^{2} / U_{9,0}^{2}\left(\theta_{0}, \phi_{0}\right) \eta M N(47)
$$

so that:

$$
\left|U_{0}\left(\theta_{0}, \phi_{0}\right)\right|^{2}=\left|U_{0,0}^{2}\left(\theta_{0}, \phi_{0}\right)\right|^{2}\left[\sum_{m=1}^{M} \sum_{n=1}^{N} a_{m m}\right]^{2}
$$

Therefore, we can write equation (43) as (Ref. 1):

$$
\begin{aligned}
\left.\overline{U(\theta, \phi)}\right|^{2}= & \left|U_{0}(\theta, \phi)\right|^{2}+\left|U_{0}\left(\theta_{0}, \phi_{0}\right)\right|^{2} \\
& \times\left[\left[U_{0.0}^{2}(\theta, \phi) \varepsilon^{2}(\theta, \phi)\right]\right. \\
& \left.+\left[U_{\theta, 0}^{2}\left(\theta_{0}, \phi_{0}\right) \eta M N P\right]\right]
\end{aligned}
$$

Allen states that:

We have previously seen that the percentage pattern error near the beam maximum is trivial, or else the Central Limit Theorem is not applicable. Further, If the array is large and the correlation interval of the far-field is small, we would expect that the total radiated power would vary minutely from the average for nearly all such arrays. Thus, one would expect that a directivity value derived using (84) as the actual power density distribution over all space would be a satisfactory approximation. (Ref. 1)

Allen is speaking from the context of his paper where he has shown that the percentage pattern error near the main beam is trivial. The equation number (84) is the same, in form, as equation (49) presented in this paper.

The main beam directivity is given by: 


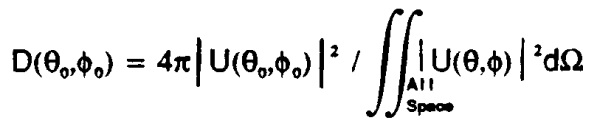

where:

$$
\mathrm{d} \Omega=\sin \theta \mathrm{d} \theta \mathrm{d} \phi
$$

Substituting equation (49) into equation (50), we find:

$$
\begin{aligned}
\mathrm{D}\left(\theta_{0}, \phi_{0}\right) & =4 \pi\left|\mathrm{U}_{0}\left(\theta_{0}, \phi_{0}\right)\right|^{2} \\
\bullet & {\left[\iint\left|\mathrm{U}_{0}\left(\theta_{, \phi}\right)\right|^{2} \mathrm{~d} \Omega+\left[\left|\mathrm{U}_{0}(\theta, \phi)\right|^{2} / \eta M N P\right]\right.} \\
\times & \left.\iint \varepsilon^{2}(\theta, \phi)\left[U_{0,0}^{2}(\theta, \phi) / U_{0.0}^{2}\left(\theta_{0}, \phi_{0}\right)\right] \mathrm{d} \Omega\right]
\end{aligned}
$$

In deriving equation (52) it was assumed that the statistical side lobe level can be neglected compared to the peak intensity so that the second term in equation (49) can be disregarded in the numerator of equation (52)(Refs. 1;22). We can denote the no-error directivity as $\mathrm{D}_{0}\left(\theta_{0}, \phi_{0}\right)$ and divide numerator and denominator of equation (52) by $4 \pi\left|U_{0}\left(\theta_{0}, \phi_{0}\right)\right|^{2}$ and we have:

$$
\begin{aligned}
D\left(\theta_{0}, \phi_{0}\right) & =\left[\left[1 / D_{0}\left(\theta_{0}, \phi_{0}\right)\right]+(1 / 4 \pi \eta M N P)\right. \\
& \left.X \iint^{2} \varepsilon^{2}(\theta, \phi)\left[U_{0.0}^{2}(\theta, \phi) / U_{0.0}^{2}\left(\theta_{0} . \phi_{0}\right)\right] d \Omega\right]-1
\end{aligned}
$$

Before we proceed. let us consider the angle dependence of $\varepsilon^{2}(\theta, \phi)$. We remember that $A(\theta, \phi)$ depends upon $\sigma_{A}^{2}(\theta, \phi)$ which contains two terms:

$$
\sigma_{A}^{2}(\theta, \phi) \approx \sigma_{\mu}^{2}(\theta, \phi)+\sigma_{\Delta}^{2}
$$

The term $\sigma_{\Delta}^{2}$ is angle invariant. However. note that the term $\sigma_{n}^{2}(\boldsymbol{\theta}, \phi)$, the element factor variance about its average, may vary with angle and may well become larger, as $U_{,}(\theta, \phi)$ becomes small (in the side lobe region of the element) (Ref. 1). Now we need to examine the angle dependence of $\Psi_{A P}(\theta, \phi):$

$$
\begin{gathered}
\Psi_{A P}(\theta, \phi)=\exp \left[-\left[\overline{\delta^{2}}+k^{2}\left(\overline{\gamma_{x}^{2}} \sin ^{2} \theta \cos ^{2} \phi\right.\right.\right. \\
\left.\left.\left.+\overline{\gamma_{y}^{2}} \sin ^{2} \theta \sin ^{2} \phi+\overline{\gamma_{z}^{2}} \cos ^{2} \theta\right)\right]\right]
\end{gathered}
$$

where, again, $\overline{\gamma_{x}^{2}}, \overline{\gamma_{y}^{2}}$, and $\overline{\gamma_{2}^{2}}$ are the variances in element radiation center location. Allen notes that, if all the variances are roughly equal, then the angle dependence of $\Psi_{A P}(\theta, \phi)$ vanishes and we can write (Ref. 1):

$$
\Psi_{A \mathrm{P}}=\exp \left[-\left(\overline{\delta^{2}}+k^{2} \overline{\gamma_{x}^{2}}\right)\right]
$$

All the angular dependence of $\varepsilon^{2}$ has now been considered.

Thus, $\varepsilon^{2}(\theta . \phi)$ is only weakly angle dependent (Ref. 1) and we can write (Ref. 1):

$$
\begin{aligned}
(1 / 4 \pi) \iint\left[U_{0.0}^{2}(\theta, \phi) / U_{0,0}^{2}\left(\theta_{0}, \phi_{0}\right)\right] d \Omega \\
\quad=\left[4 \pi U_{\theta_{0} .}^{2}\left(\theta_{0}, \phi_{0}\right) / \iint U_{\theta .0}^{2}(\theta, \phi) d \Omega\right]-1 \\
\quad=\left[d\left(\theta_{0}, \phi_{0}\right)\right]^{-1}
\end{aligned}
$$

where $d\left(\theta_{0}, \phi_{0}\right)$ is the element directivity in the $\theta_{0}, \phi_{0}$ direction. Hence:

$$
\begin{aligned}
\mathrm{D}\left(\theta_{0}, \phi_{0}\right)= & {\left[1 / \mathrm{D}_{0}\left(\theta_{0}, \phi_{0}\right)\right.} \\
& \left.+\varepsilon^{2} /\left[\mathrm{d}\left(\theta_{0}, \phi_{0}\right) \eta M N P\right]\right]^{-1}
\end{aligned}
$$

Allen states we can write the no-error array directivity as (Rөf. 1):

$$
D_{0}\left(\theta_{0} ; \phi_{0}\right)=d\left(\theta_{0} ; \phi_{0}\right) \eta M N
$$

assuming an array with at least 25 elements (Ref. 1). Thus we can write our final result as (Ref. 1):

$$
D\left(\theta_{0}, \phi_{0}\right)=D_{0}\left(\theta_{0}, \phi_{0}\right)\left[1+\left(\varepsilon^{2} / P\right)\right]^{-1}
$$




\section{PROGRAM MECHANICS}

\section{Program Development}

In this section. the development of the main program and the subprograms used to analyze the performance of a planar array. The overall program is subdivided into five functional parts: (1) Data entry and element excitation routines; (2) Design and expected radiation pattem calculations; (3) Design and expected beamwidth calculations; (4) Directivity calculations; and (5) Output routine and recalculation.

Part 1 - Data Entry and Element Excitation Routines

The program begins by asking the user for the design detalls of the planar array. The user is allowed to enter the center operating frequency or wavelength, a bandwidth of operation, array specifications in the $x$-direction (number of elements, interelement spacing, and type of excitation), and the array specifications in the y-direction. For element excttations. the user may choose between Dolph-Chebyshev synthesis. Taylor $n$-parameter synthesis, a userdefined current distribution, Binomial current distribution, or a Uniform current distribution for the $x$ - and $y$-directions, independently. If the Dolph-Chebyshev synthesis is chosen. the user enters the desired side lobe level and the program computes the element weights based on an algorithm presented by Bresler (Ref. 5) and recommended by Bums, Laxpati. and Shelton (Ref. 6). If the Taylor $n$-parameter synthesis is chosen, the user enters the desired side lobe level and the $n$-parameter and the program computes the element weights following an algorithm written by Pozar (Ref. 19) which is based on the works of Elliott (Ref. 12) and Balanis (Ref. 4). The program returns element weights calculated using the Null Matching method (Ref. 12) and the Aperture Sampling method (Ref. 4). The user chooses which set of weights to continue with in the program. A complete discussion of the expressions used in the element weight calculation portion of the program can be found in (Ref. 8).
After the array specifications are entered. the user selects the orientation and the length of the dipole to be used in the analysis. The user has four choices: (1) a dipole oriented in the $x$-direction, (2) a dipole oriented in the $y$ direction. (3) a dipole oriented in the $z$-direction. or, even though not a dipole, (4) an isotropic element.

Next. the user enters the tolerance data for the planar array. The user first enters the Root-Mean-Square (RMS) amplitude error. Referring to equation (17), this is the term " $\sigma_{\Delta}$ " which is the standard deviation while the term " $\sigma_{\Delta}^{2 n}$ is the variance or mean square value. Next. the user enters the RMS phase error in degrees. This term is converted to become the

term $\overline{\delta^{2}} "$ in equation (37). Then in succession. the user enters the RMS error in the $x, y$, and $z$ placement of the element. These three terms

become $\overline{\gamma_{x}^{2}}, \overline{\gamma_{y}^{2}}$, and $\overline{\gamma_{z}^{2}}$, respectively, in equation (17). Lastly, the user enters the fraction of elements actually operating (the variable "P" in equation (18)) in the array.

The last item the user enters are the scan angles, $\theta_{0}$ and $\phi_{0}$. These angles are shown in Figure 2.

\section{Part 2 - Radiation Pattern Calculations}

The second part of the program deals with generating the pattern plots for the design and expected arrays. The program calculates the data points necessary to obtain pattern cut plots in the $\theta_{h}$ and $\Psi_{h}$ planes as shown in Figure 2. The $\theta_{h}$ plane is defined as the plane in the $\theta$ direction. The $\Psi_{h}$ plane is the plane perpendicular to the $\Theta_{h}$ plane. The most efficient way to obtain these pattern cuts is to define a new coordinate system that aligns with the direction of the main beam. such that. the $z$-axis is, once again, aligned along the boresight of the main beam as it would be for a broadside array. 
We wish to express the spherical angle $\theta$ and $\phi$ in terms of $\theta^{\prime}$ and $\phi^{\prime}$ with the pointing angles $\theta_{0}$ and $\phi_{0}$ supplied by the user. This geometry is shown in Figure 3. The angles $\theta_{0}$ and $\phi_{0}$ are Eulerian angles that produce two rotations in the cartesian coordinate system to align a new primed cartesian coordinate system along the main beam (Ref. 15). Once this translation takes place, the pattem cuts can be taken in the $x^{\prime} z^{\prime}-$ and $y^{\prime} z^{\prime}$-planes representing the $\Theta_{n}$ - and $\Psi_{n}$-plane pattern cuts, respectively, as shown in Figure 4. Performing the translation produces new expressions for $\sin \theta \cos \phi$, $\sin \theta \sin \phi$, and $\cos \theta$ in terms of $\theta^{\prime} . \phi^{\prime}, \theta_{0^{*}}$ and $\phi_{0}$ which are substituted into equations (1) and (39). These new expressions are:

$$
\begin{aligned}
& \sin \theta \cos \phi= \sin \theta^{\prime}\left(\cos \phi_{0} \cos \theta_{0} \cos \phi^{\prime}-\sin \phi_{0} \sin \phi^{\prime}\right) \\
&+\cos \phi_{0} \sin \theta_{0} \cos \theta^{\prime} \\
& \sin \theta \sin \phi= \sin \theta^{\prime}\left(\sin \phi_{0} \cos \theta_{0} \cos \phi^{\prime}+\cos \phi_{0} \sin \phi^{\prime}\right) \\
&+\sin \phi_{0} \sin \theta_{0} \cos \theta^{\prime} \\
& \cos \theta=-\sin \theta_{0} \sin \theta^{\prime} \cos \phi^{\prime}+\cos \theta_{0} \cos \theta^{\prime}
\end{aligned}
$$

The user is given the option of generating up to 12 different sets of data to later produce 12 different plots. The user is asked if data points for the design and expected radiation patterns are desired at the center frequency or wavelength of the operating band. If requested. four sets of data are generated, two sets for the design pattern ( $x^{\prime} z^{\prime}-$ and $y^{\prime} z^{\prime}-$ planes) and two sets for the expected pattern ( $x^{\prime} z^{\prime}-$ and $y^{\prime} z^{\prime}$-planes). After these data sets are calculated. the user is asked if data sets are wanted for the lower frequency and then the upper frequency of the bandwidth.

\section{Beamwidth Calculations}

Generally, the task of finding the beamwidth of a planar array is quite difficult. However, with the aid of a computer and the aforementioned coordinate translation, this task is greatly simplified as shown next. The following method is accurate for any array scanned in any direction.

Consider the $\left(x^{\prime}, y^{\prime}, z^{\prime}\right)$ coordinate system as shown in Figure 3. Looking in the $x^{\prime} z^{\prime}-$ plane, the program begins its calculation of the

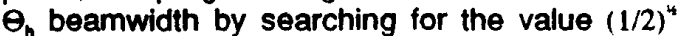
where $\phi^{\prime}$ is equal to $180^{\circ}$ by varying $\theta^{\prime}$ in one degree increments until the magnitude of the pattern is equal to just less than $(1 / 2)^{*}$. If the magnitude is equal to $(1 / 2)^{4}$ the angle is stored $\theta^{\prime}$ left and the routine begins searching where $\phi^{\prime}$ is equal to $0^{\circ}$. If the magnitude of the pattern is just less than $(1 / 2)^{4}$ then that angle and the one just prior are sent to a routine that solves for the half-power point using a secant root finding algorithm. This algorithm locates the angle to within a tolerance of $1.0\left(10^{-7}\right)$ of $(1 / 2)^{4}$. The angle produced by the secant routine is then stored as $\theta^{\prime}$ left. This same process is repeated for $\phi^{\prime}=0^{\circ}$ and this angle is stored as $\theta^{\prime}$ right. Then the bearmwidth $\Theta_{h}$ is found by adding $\theta^{\prime}$ left and $\theta^{\prime}$ right. A similar process is followed in finding the beamwidth $\Psi_{h}$. The angle $\phi^{\prime}$ is set to $270^{\circ}$ and $\theta^{\prime}$ varied in one degree increments until the magnitude of the pattern is equal to or just less than $(1 / 2)^{4}$. The root finding algorithm is called if the magnitude does not equal $(1 / 2)^{n}$ and a value returned for $\psi$ left. The same process is followed in locating $\psi$ right where $\phi^{\prime}$ equals $90^{\circ}$. The beamwidth $\Psi_{h}$ is found by adding $\psi$ left and $\psi$ right. The halfpower beamwidths for the expected array are found in the same fashion. The design and expected beamwidths are calculated for the upper, center, and lower frequencies of the bandwidth.

\section{Part 4 - Directivity Calculations}

The directivity of a planar array $\left(D_{n}\right)$ is defined as the maximum value of directive gain or (Ref. 22):

$$
D_{0}=4 \pi U\left(\theta_{0}, \phi_{0}\right) / \Omega_{\Lambda}
$$

where $\Omega_{A}$ is the beam solid angle defined by:

$$
\Omega_{\wedge}=\iint_{\Omega}|U(\theta, \phi)|^{2} \mathrm{~d} \Omega
$$


and

$$
d \Omega=\sin \theta d \theta d \phi
$$

Evaluating the integral in equation (63) would involve a significant amount of computer time. Instead. there is an approximation for directivity which is accurate provided reasonable excitation schemes are used, the element pattem is such to eliminate the total pattem in the half space $\theta>\pi / 2$ but is broad enough to be ignored in the half space $\theta \leq \pi / 2$, and the array is not scanned closer than several beamwidths of endfire.

The directivity of a planar array can be calculated using (Ref. 10):

$$
D_{0}=\pi \cos \theta_{0} D_{x} D_{y}
$$

where $D_{x}$ and $D_{y}$ are the directivities of two broadside arrays in the $x$ and $y$ directions, respectively. Equation (65) is used in the program to calculate the directivity of a planar array. Equation (66) is used to calculate $D_{x}$ and $D_{y}$ in equation (65)(Ref. 23):

$$
\begin{aligned}
& D_{f}=\left[\sum_{m=1}^{M} A_{m}\right]_{2} \\
& \left.+\left[\sum_{m=1}^{M} \sum_{m=1}^{M} A_{m} A_{p} \mid \sin \left[(m-p) k d_{j}\right) /(m-p) k d_{j}\right] \mid\right]
\end{aligned}
$$

which is valid for a broadside, equally spaced array.

Equation (65) is sufficient for dipole elements up to approximately one wavelength in length. As the length of the dipole increases beyond one wavelength $(L>\lambda)$, the number of lobes begin to increase and the main lobe becomes narrower, thus negating the assumption that the element pattem is broad.

The change in directivity due to errors is calculated by the program using equation (58) modified as follows:

$$
\Delta D=\left[1+\left(\varepsilon^{2} / P\right)\right]^{-1}
$$

Linear arrays and individual dipoles can also be considered by the program, and code was included to calculate the directivity of each. A complete discussion of the expressions used to calculate the directivity of the linear array or the dipole are presented in (Ref. 8)

Recalculation 5 - Output Routine and

All the data collected up to this point is sent to an output routine. A sample of the output file is shown in Figure 5. After the data is sent to the output file, the user is prompted for instructions on how to proceed. The user may terminate the program or recalculate new patterns, beamwidths, and directivities for a new set of scan angles.

\section{PROGRAM VALIDATION}

A great deal of effort went in to validating all the code used in the program. However, in the interests of brevity, only a few representative cases are shown in this paper that were used to validate the beamwidth, directivity, and error routines. The interested reader is referred to (Ref. 8) for all the details in validating this program.

\section{Validation of the Beamwidth and Directivity Calculation Routines}

The first part of this section deals with validating the beamwidth calculation routines in the program. First the program is validated against several linear array examples and then two planar array examples found in the literature. The last part of this section validates the directivity calculation routines against the same cases as for the beamwidth validation.

\section{Beamwidth Validation}

Eight different linear array cases found 
in the literature are used to validate the beamwidth routines. The eight linear cases are:

Case 1: Uniform current distribution, 5 elements, $\lambda / 2$ spacing, $\theta_{0}=0^{\circ}$, isotropic elements (Ref. 23).

Case 2: Triangular current distribution. 5 elements, $\lambda / 2$ spacing, $\theta_{0}=0^{\circ}$, isotropic elements (Ref. 23).

Case 3: Inverse triangular current distribution, 5 elements, $\lambda / 2, \theta_{0}=0^{\circ}$, isotropic elements (Ref.23).

Case 4: Binomial current distribution. 5 elements, $\lambda / 2$ spacing, $\theta_{0}=0^{\circ}$, isotropic elements (Ref. 23).

Case 5: Dolph-Chebyshev synthesis, $-20 \mathrm{~dB}$ design side lobe level, 5 elements, $\lambda / 2$ spacing, $\theta_{0}=0^{\circ}$, isotropic elements (Ref. 23).

Case 6: Dolph-Chebyshev synthesis, $-30 \mathrm{~dB}$ design side lobe level, 5 elements, $\lambda / 2, \theta_{0}=0^{\circ}$, isotropic elements (Ref. 23).

Case 7: Dolph-Chebyshev synthesis, $-26 \mathrm{~dB}$ design side lobe level, 10 elements, $\lambda / 2, \theta_{0}=0^{\circ}$, isotropic elements (Ref. 4).

Case 8: Taylor $\bar{n}$-parameter synthesis $(\bar{n}=6)$, -20 dB design side lobe level. null matching weights, 19 elements, $0.7 \lambda$ spacing, $\theta_{0}=0^{\circ}$, isotropic elements (Ref. 19).

The beamwidths found in the literature and as calculated by the program are presented in Table I for comparison. Comparing the beamwidths, we can see excellent agreement, except in Case 7. In Case 7, the beamwidths calculated by Balanis are calculated using certain approximations which may not be as accurate as the program.

Now, let us look at two cases for planar arrays and compare results. The two planar array cases are:
Case 9: A 10x10 array, Dolph-Chebyshev synthesis in both directions, $-26 \mathrm{~dB}$ design side lobe level, $\lambda / 2$ spacing both directions, $\theta_{0}=30^{\circ}$, $\phi_{0}=45^{\circ}$, isotropic elements (Ref. 4).

Case 10: A 20×36 array, Dolph-Chebyshev synthesis both directions. $-30 \mathrm{~dB}$ design side lobe level, $0.58 \lambda$ spacing in the $x$-direction. $0.64 \lambda$ spacing in the $y$-direction, $\theta_{0}=0^{\circ}, \phi_{0}=0^{\circ}$. isotroplc elements (Ref. 10).

The beamwidths for the planar array cases found in the literature and as calculated by the program are presented in Table II for comparison. Again, there is good agreement between program results and the examples in the literature. Any discrepancies can be attributed to approximations used by the various authors.

\section{Directivity Validation}

The same ten cases used to validate the beamwidth calculations are used to validate the directivity calculation routines. The directivities found in the literature and those calculated by the program are presented in Table III for comparison. Again we see excellent agreement with any discrepancies attributed to accuracy of element weights and approximations used by the various authors.

\section{Error Validation}

In this section, we will validate the error routines by confirming various trends in tolerance analysis noted in the literature. Unfortunately, specific examples for either the linear array or the planar array are not presented in the literature. If the following trends are confirmed. then, we will have reasonable confidence that the error routines are performing properly.

Before the trends are confirmed. we will run the program for a no-error situation to ensure the beamwidths of the no-error error routines match those of the design routines and to show that the change in directivity is $0 \mathrm{~dB}$. We will rerun Case 7 to ensure the beamwidths 
of the no-error error routines match those of the design values. Table IV shows excerpts from the output file. We can see that the design and expected beamwidths match precisely and that the change in directivity is $0 \mathrm{~dB}$. Therefore, any differences we see between the design and expected values are due to errors in the design and not numerical errors caused by the program.

Five different trends were identified during the literature review and will be used to validate the error routines. The five trends are:

Trend 1: The rise in side lobe level due to random errors, for a given set of tolerances and number of elements, increases as the side lobe level is further suppressed (Refs. $3 ; 11 ; 18$ ).

Trend 2: For a given set of tolerances, pattern deterioration is found to decrease as the array is enlarged (Reis. $11 ; 20$ ).

Trend 3: For a given set of tolerances, pattem deterioration is less for a planar array of size $L^{2}$ than it is for a linear array of length $L$ (Ref. 11).

Trend 4: The side lobe level increase due to random errors does not depend on scan angle (Ref. 11).

Trend 5: Translational errors in the positions of the elements are found to cause the dominant effect while amplitude errors in the radiating currents are of secondary importance (Ref. 11).

When Elliott showed the fifth trend. he did not consider the fraction of elements operating. Therefore, when we confirm the fifth trend, we will set the fraction of elements operating to one and then proceed. The fraction of elements operating will overshadow any other errors in the design as we will show in confirming the fifth trend.

All five trends will be confirmed by using Dolph-Chebyshev synthesized arrays with isotropic elements. Dolph-Chebyshev patterns provide a good pattern for analyzing these trends. Also, isotropic elements are used since dipoles tend to distort the pattem and what we really want to see are pattern distortions due to errors. Since we will be using isotropic elements. the RMS error in the element pattern will be set to $-100 \mathrm{~dB}$ so as not to have any effect on the calculations.

To confirm the first trend. let us consider a $10 \times 10$ element planar array. with a DolphChebyshev synthesized current distribution in both directions and $\lambda / 2$ element spacing. Four runs of the program were made with a decrease of $5 \mathrm{~dB}$ in the side lobe level for each run beginning with a $-25 \mathrm{~dB}$ design side lobe level. The following error data was used for each run:

RMS amplitude error

0.002 units

RMS phase error:

10.0 degrees

RMS error in x-placement: $\quad 0.002 \mathrm{cms}$

RMS error in y-placement: $\quad 0.002 \mathrm{cms}$

RMS error in z-placement: $\quad 0.002 \mathrm{cms}$

RMS error in element pattern: $-100.0 \mathrm{~dB}$

Fraction of elements operating: $\quad 1.0$

The plots of the expected radiation pattern overiaid on the design radiation pattern for each run are shown in Figures 6-9. Comparing these figures, we can see the side lobe level of the error pattem rising the further the design side lobe level is suppressed confirming the first trend. This trend can be explained by comparing the element weights for each run. As the side lobe level is further reduced, the end element weights begin to increase causing an abrupt change in the aperture distribution at the ends of the array. This abrupt change at the ends of the array coupled with the errors in the design cause the side lobe level to increase.

The second trend is confirmed by considering four different sizes of planar arrays. Each array will have a Dolph-Chebyshev synthesized current distribution with a design side lobe level of $-30 \mathrm{~dB}$ and the same errors presented earlier. Figures 10-13 show the expected radiation pattem overlaid on the design radiation pattern for a $10 \times 10,12 \times 12,15 \times 15$, and $20 \times 20$ element planar array, respectively. By 
comparing these figures, we see the pattern distortion decreasing as the size of the array increases confirming the second trend. The reason this trend is occurring is because as the array becomes larger (more elements) the sum of all the errors tends to the mean of the design thus reducing the overall effects of the errors.

For the third trend. we will consider a $20 \times 20$ element planar array and a 20 element linear array. Each array will have a DolphChebyshev synthesized current distribution giving a $-30 \mathrm{~dB}$ design side lobe level, $\lambda / 2$ element spacing, and the same set of tolerances used earlier. The expected radiation pattern overlaid on the design radiation pattem is shown in Figure 14 for the planar array. The expected radiation pattern overlaid on the design radiation pattern for the linear array is shown in Figure 15. Comparing Figures 14 and 15 we can see that the expected pattem in Figure 14 is-considerably less distorted than the expected pattern in Figure 15. Again, the reason this trend occurs can be attributed to the increased number of elements and the fact that the errors tend to their mean value as the population (of elements) grows.

The fourth trend is confirmed by considering five different scan angles in the same array. The scan angle is varied. in $10^{\circ}$ increments beginning at $0^{\circ}$ and scanning through $40^{\circ}$ for a $15 \times 15$ element planar array with a Dolph-Chebyshev synthesized current distribution giving a $-30 \mathrm{~dB}$ design side lobe level, with $\lambda / 2$ element spacing. The case for the array scanned at broadside $\left(0^{\circ}\right)$ is shown in Figure 48. The cases for the array scanned at $10^{\circ}, 20^{\circ}, 30^{\circ}$, and $40^{\circ}$ are shown in Figures $16-$ 19, respectively. Each figure shows the expected radiation pattern overlaid on the design radiation pattern. By comparing all five figures, we can see that the side lobe level due to errors remains constant as the array is scanned through $40^{\circ}$ confirming this trend. This trend is best explained by considering equation (39) and the same reasoning used in deriving the change in directivity due to errors. In the Random Error Effects on Array Directivity section we showed that $\varepsilon^{2}(\theta, \phi)$ was only weakly angle dependent and, this being the case, the second term in equation (39) becomes that of an omnidirectional type pattern. This adds a constant term to the overall pattem that does not increase or decrease with scan angle.

In order to validate the fifth trend an extensive sensitivity analysis would have to be undertaken which is not within the purview of this paper. Instead, we will take a qualitative look at which errors are the most dominant by examining equation (39), and by looking at a pattern with typical errors, and removing them one-at-a-time until the design pattern is obtained.

For this analysis, the absolute value of

$\overline{U(\theta, \phi)}^{2}$ is immaterial so we can divide equathon (39) by $P=\Psi_{A P}(\theta, \phi)$ and look at the term preceding the double summation of the second term in equation (39). Calling this term the error term (E.T.) we have:

$$
E_{.} T_{0}=\left[\left[1+\sigma_{A}^{2}(\theta, \phi)\right] / P \Psi_{A P}(\theta, \phi)\right]-1
$$

If the variances in the term $\Psi_{A p}(\theta . \phi)$ as given by equation (37) are roughly equal, we can rewrite equation (68) as:

$$
\text { E.T. }=\left[\left[1+\sigma_{A}^{2}\right] \exp \left(\bar{\delta}^{2}\right) \exp \left(k^{2} \bar{\gamma}_{x}^{2}\right) / P\right]-1
$$

In equation (69) we see that the error term is inversely proportional to the fraction of elements operating. The variable $P$ clearly has the potential for causing the dominant effect if element reliability is not very high. Assuming for now that $P$ is approximately one the next dominant effect will come from the translational errors in the positions of the elements. Since the variance in the element position is multiplied by the factor $\mathbf{k}^{2}$ in the exponential. it clearly has the leading effect on the overall pattem. It is purely conjecture as to which of the remaining two factors, variance in the phase error or the amplitude error, would be the more dominant of the two. Elliott claims that errors in 
the radiating currents are of secondary importance (Ref. 11).

To illustrate which errors are most dominant and the least dominant, let us consider a case with realistic errors. Consider a $10 \times 10$ planar array with a Dolph-Chebyshev synthesized current distribution giving a $-30 \mathrm{~dB}$ design side lobe level and $\lambda / 2$ element spacing. The center operating frequency is $18 \mathrm{GHz}$. For the errors, we will assume the radiating elements are tolerably matched to the feed structure such that the RMS amplitude and phase errors are 0.1 and $5^{\circ}$, respectively. We will assume the array is manufactured with a 0.05 centimeter RMS error in the $(x, y, z)$ placement of the individual radiating elements. Finally, for the mission being considered, we can expect no more than 10 elements will fall. The resulting total error pattern overlaid with the design pattem is shown in Figure 20. Now let us remove the errors one-at-a-time until we get the design radiation pattem back. With the phase error set to zero, the resulting pattern is shown in Figure 21. Comparing Figures 20 and 21 we see very little change in the expected pattern. The expected pattern with both the amplitude and phase errors set to zero and all other errors remaining is shown in Figure 22. Again. there is very little change in the expected pattem. The expected pattern with the fraction of elements set to one and the amplitude and phase errors set to zero and just the translational errors remaining is shown in Flgure 23 . We can see a significant change in the expected patterns between figures 22 and 23 . Setting the translational errors to zero so that all errors are zero or one in the case of the fraction of elements operating, we obtain the design pattern as shown in Figure 24. Though not conclusive, we can infer that the translational errors, as small as they were, caused the largest degradation in the pattern as evidenced by comparing the transition of the expected pattern from Figure 22 to 23 and then from Figure 23 to 24 . Of secondary importance is the fraction of elements operating followed by the amplitude error.

\section{CONCLUSIONS}

A program was developed to analyze the effects of perturbations in an array's design parameters on the overall performance of the array. An expression relating these perturbations to the planar array's radiation pattern was derived and used in developing the tolerance analysis routines used in the program. Tolerances considered by the program include amplitude and phase errors in the element's drive current, errors in the element positions within the array, and errors between an element's actual pattern and the average element pattern. as well as the fraction of elements operating. The program user can input an array's design parameters and tolerances and assess the impact of the tolerances on the array's side lobe level. beamwidth, and directivity for a specified bandwidth and scan angle.

Several trends were evident as confirmed by the program. First. the rise in side lobe level due to random errors, for a given set of tolerances and number of elements, increases (relative to the design side lobe level) as further design side lobe level suppression is attempted. Second, for a given set of tolerances, pattern deterioration was found to decrease as the array was enlarged. Third, for a given set of tolerances, pattern deterioration is less for a planar array of size $L^{2}$ than it is for a linear array of length $L$. Fourth, the side lobe level increase due to random errors does not depend on scan angle or is, at most. weakly dependent on scan angle. Finally. translational errors in the positions of the elements were found, qualitatively, to cause the dominant effect in pattem deterioration provided the fraction of elements operating is high.

The array antenna engineer or program manager now has a useful toot for assessing the effects of tolerances, at least to a first order, on the performance of an array. Two avenues are now evident for the user in designing an array in the presence of errors. The user can specify the tolerances in a specification or the user can over-design the array by designing for 
an $M d B$ side lobe level when an $N d B$ side lobe level is desired. The user can use this program to determine the amount of overdesign needed to produce the desired side lobe level.

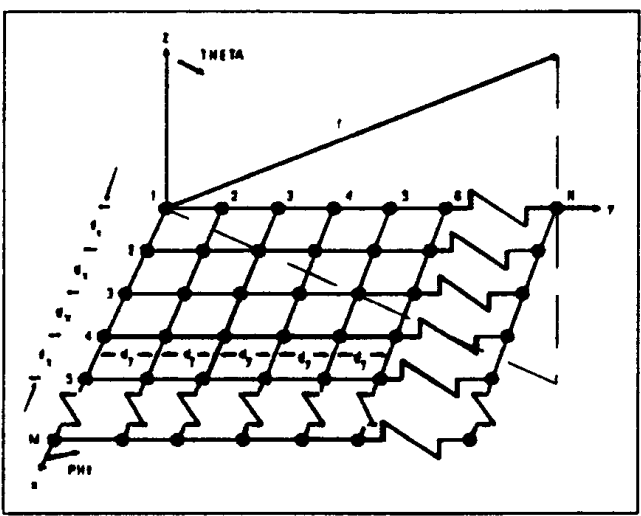

Figure 1. Planar array geometry

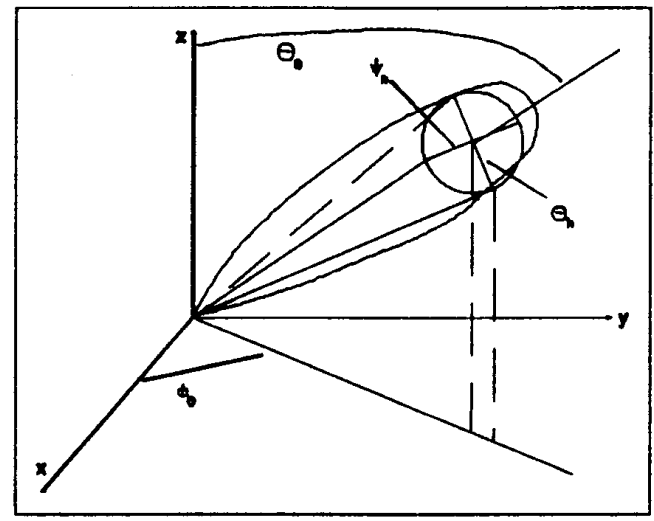

Figure 2. Half-power beamwidths for a conical beam oriented toward $\theta=\theta_{0}, \phi=\phi_{0}$

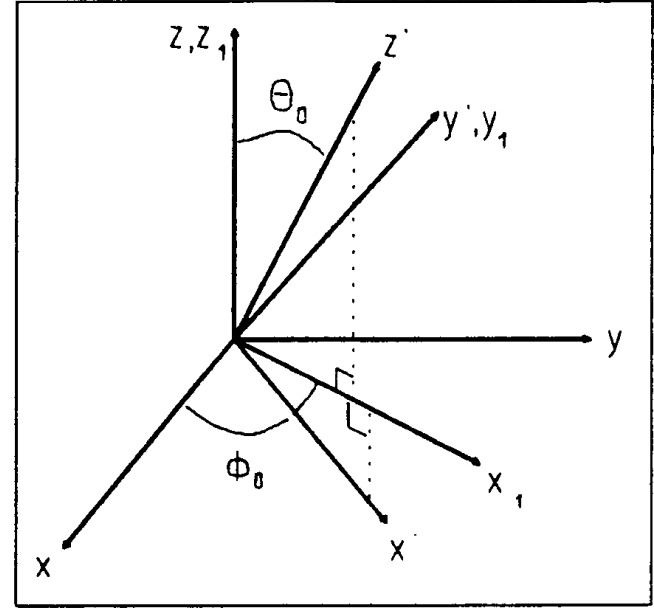

Figure 3. Geometry for beamwidth calculations

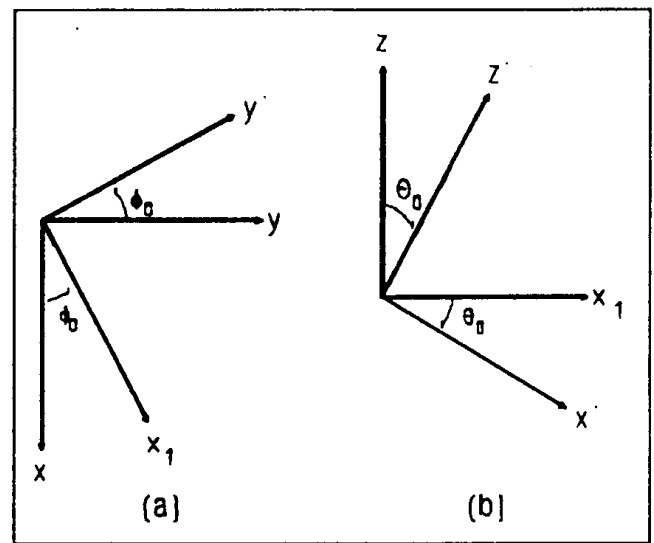

Figure 4. Coordinate translation steps for pattern and beamwidth calculations 


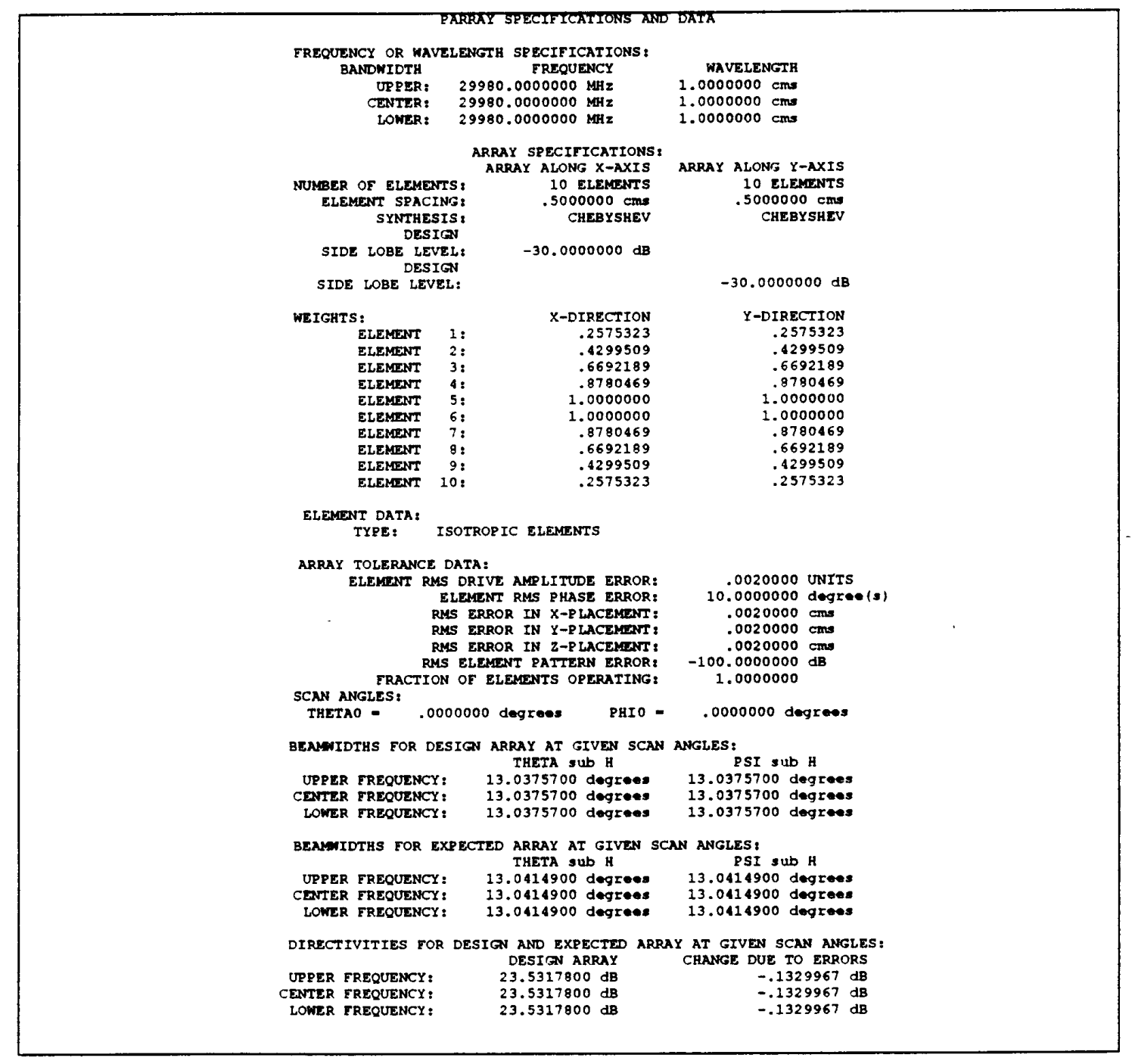

Figure 5. Sample output from planar array design analysis program 
Table I. Linear array beamwidth comparisons

\begin{tabular}{ccc}
\hline Case & Literature & Program \\
\hline 1 & $20.8^{\circ}$ & $20.7765^{\circ}$ \\
2 & $26.0^{\circ}$ & $25.95161^{\circ}$ \\
3 & $18.2^{\circ}$ & $18.23554^{\circ}$ \\
4 & $30.3^{\circ}$ & $30.28262^{\circ}$ \\
5 & $23.6^{\circ}$ & $23.70704^{\circ}$ \\
6 & $26.4^{\circ}$ & $26.40292^{\circ}$ \\
7 & $10.1^{\circ}$ & $12.34591^{\circ}$ \\
8 & $4.1^{\circ}$ & $4.128539^{\circ}$ \\
\hline
\end{tabular}

Table II. Planar array beamwidth comparisons

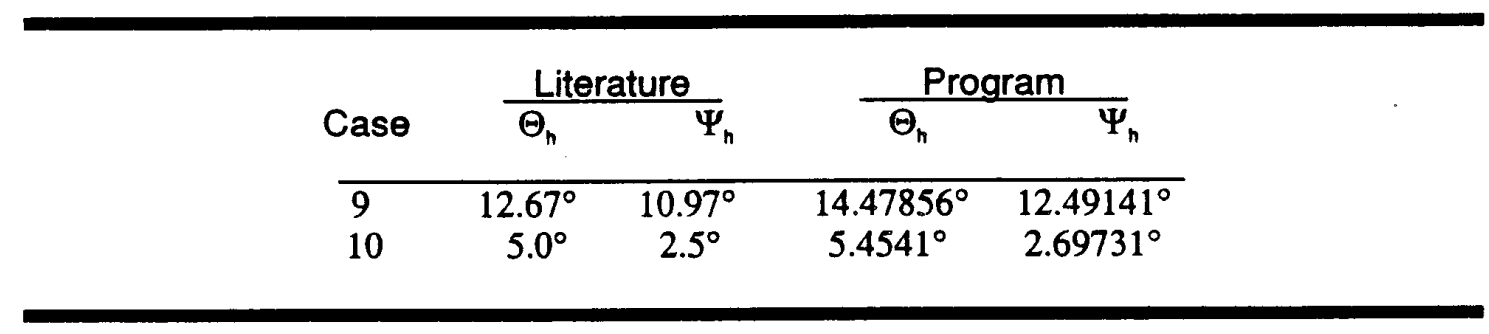


Table III. Linear and planar array directivity comparisons

\begin{tabular}{rrr}
\hline Case & Literature & Program \\
\hline 1 & $6.9897 \mathrm{~dB}$ & $6.9897 \mathrm{~dB}$ \\
2 & $6.2941 \mathrm{~dB}$ & $6.2973 \mathrm{~dB}$ \\
3 & $6.5128 \mathrm{~dB}$ & $6.5142 \mathrm{~dB}$ \\
4 & $5.6348 \mathrm{~dB}$ & $5.6314 \mathrm{~dB}$ \\
5 & $6.7025 \mathrm{~dB}$ & $6.7078 \mathrm{~dB}$ \\
6 & $6.2531 \mathrm{~dB}$ & $6.2590 \mathrm{~dB}$ \\
7 & $9.63 \mathrm{~dB}$ & $9.5074 \mathrm{~dB}$ \\
8 & $13.90 \mathrm{~dB}$ & $14.0468 \mathrm{~dB}$ \\
9 & $23.60 \mathrm{~dB}$ & $23.3615 \mathrm{~dB}$ \\
10 & 34.1 & $34.0463 \mathrm{~dB}$ \\
\end{tabular}

Table IV. Design versus no-error expected parameters

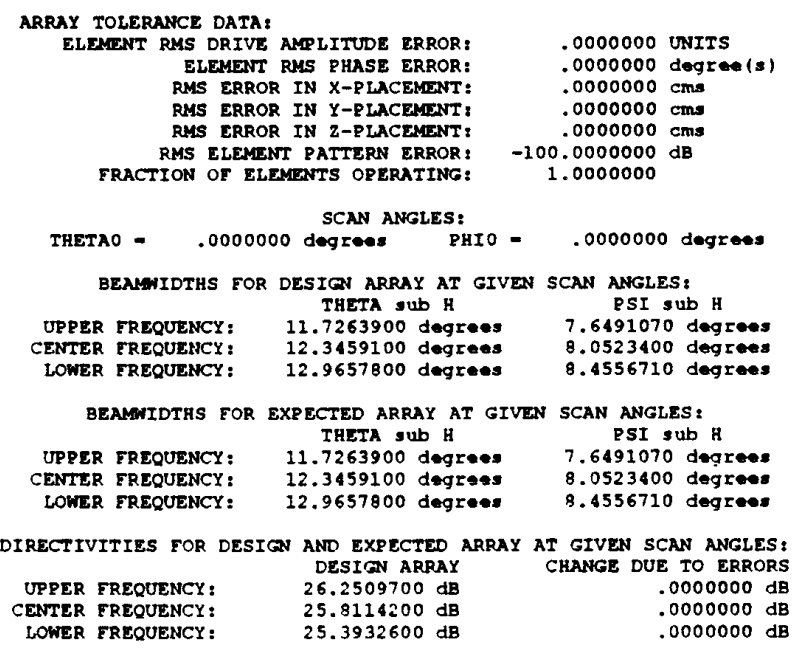




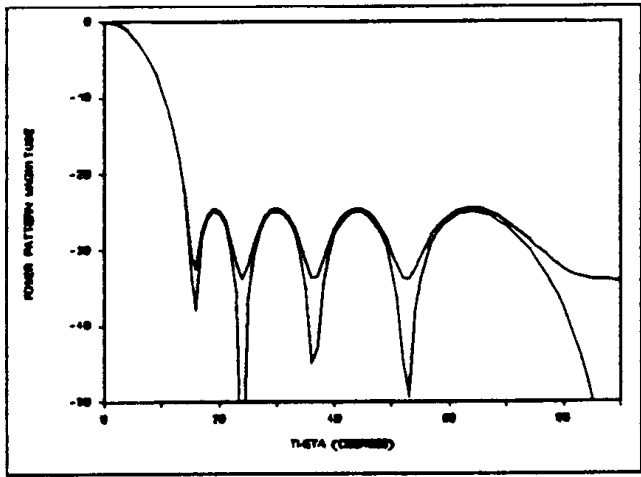

Figure 6. Expected and design radiation patterns ( $x^{\prime} z^{\prime}$-plane)for a $10 \times 10$ element, Dolph-Chebyshev systhesized array with $\lambda / 2$ spacing and $\mathrm{a}-25 \mathrm{~dB}$ design side lobe level

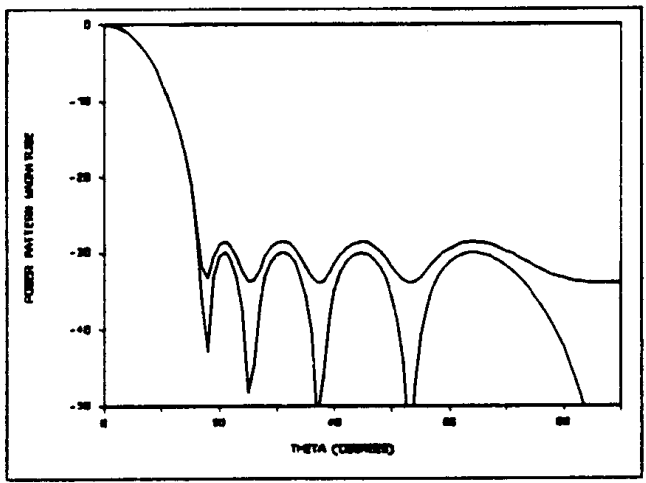

Figure 7. Expected and design radiation patterns ( $x^{\prime} z^{\prime}$-plane) for a $10 \times 10$ element, Dolph-Chebyshev systhesized array with $\lambda / 2$ spacing and a $-30 \mathrm{~dB}$ design side lobe level

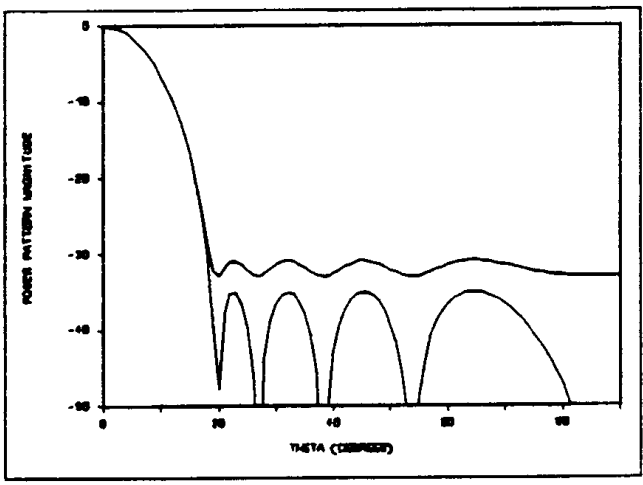

Figure 8. Expected and design radiation patterns ( $x^{\prime} z^{\prime}$-plane) for a $10 \times 10$ element. Dolph-Chebyshev synthesized array with $\lambda / 2$ spacing and $\mathrm{a}-35 \mathrm{~dB}$ design side lobe level

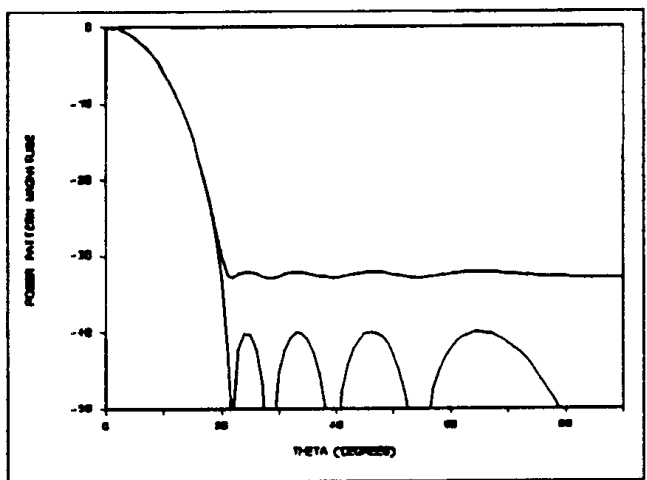

Figure 9. Expected and design radiation patterns (xz-plane) for a $10 \times 10$ element. Dolph-Chebyshev synthesized array with $\lambda / 2$ spacing and $a-40 \mathrm{~dB}$ design side lobe level 


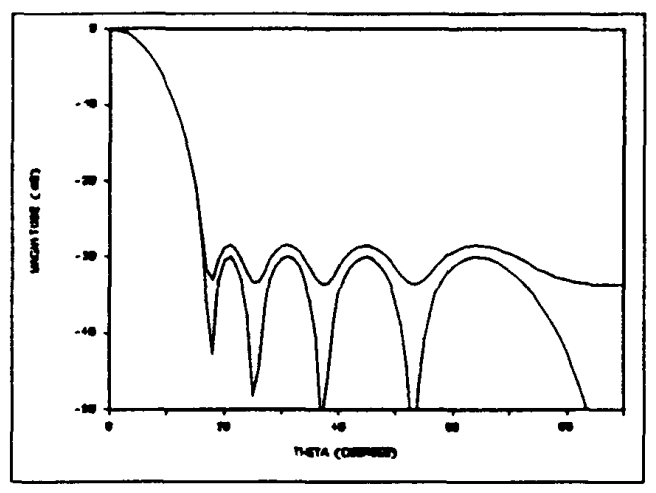

Figure 10. Expected and design radiation patterns ( $x^{\prime} z$ '-plane) for a $10 \times 10$ element, Dolph-Chebyshev synthesized array with $\lambda / 2$ spacing and a $-30 \mathrm{~dB}$ design side lobe level

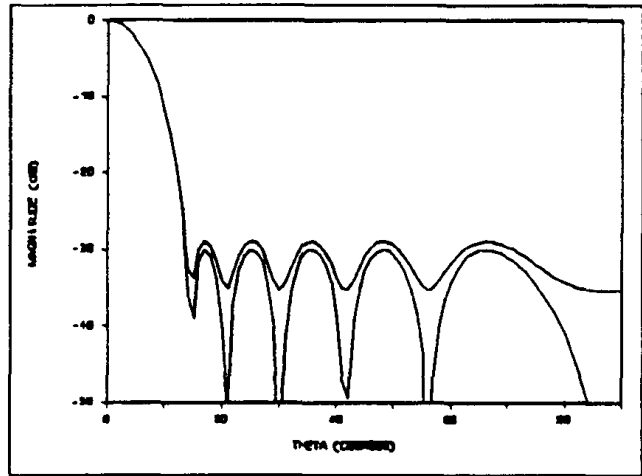

Figure 11. Expected and design radiation patterns ( $x^{\prime} z^{\prime}$-plane) for a $12 \times 12$ element, Dolph-Chebyshev systhesized array with $\lambda / 2$ spacing and a $-30 \mathrm{~dB}$ design side lobe level

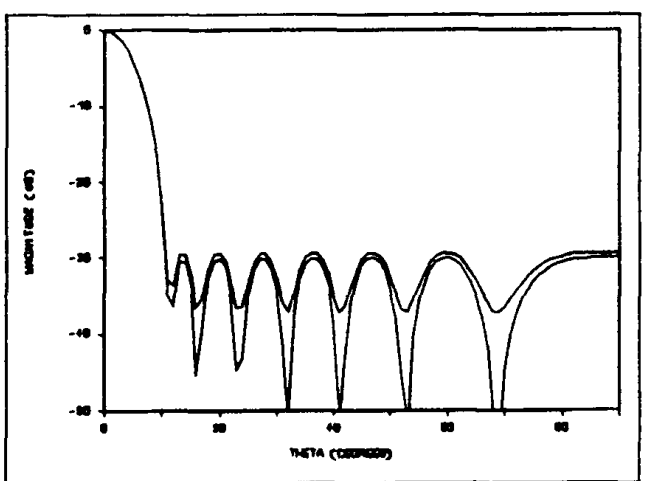

Figure 12. Expected and design radiation patterns ( $x^{\prime} z^{\prime}$-plane) for a $15 \times 15$ element. Dolph-Chebyshev synthesized array with $\lambda / 2$ spacing and a $-30 \mathrm{~dB}$ design side lobe level

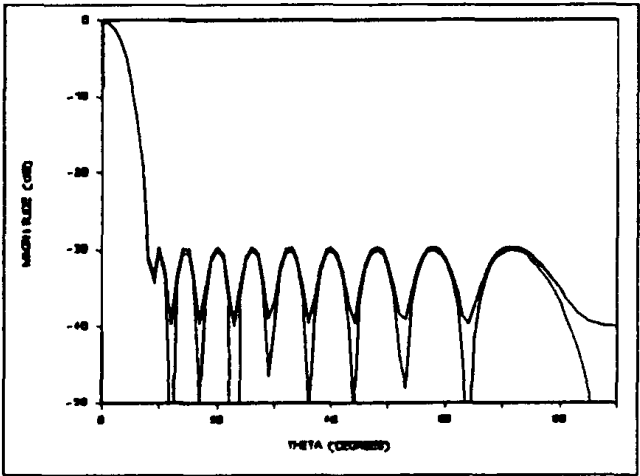

Figure 13. Expected and design radiation patterns ( $x^{\prime} z^{\prime}$-plane) for a $20 \times 20$ element. Dolph-Chebyshev synthesized array with $\lambda / 2$ spacing and $a-30 \mathrm{~dB}$ design side lobe level 


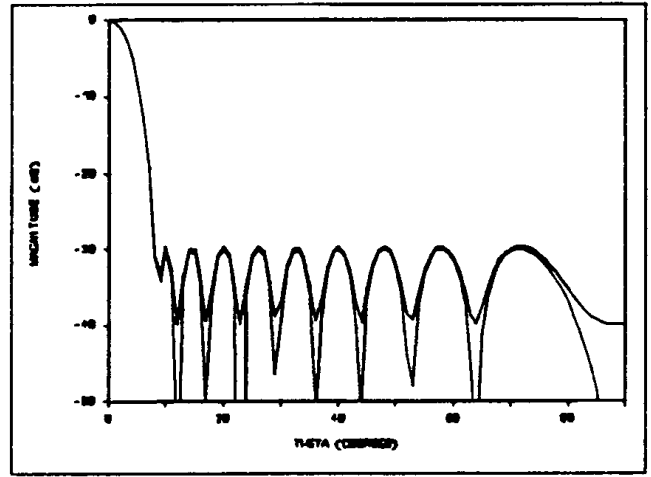

Figure 14. Expected and design radiation patterns ( $x^{\prime} z^{\prime}$-plane) for a $20 \times 20$ element, Dolph-Chebyshev synthesized array with $\lambda / 2$ spacing and a $-30 \mathrm{~dB}$ design side lobe level

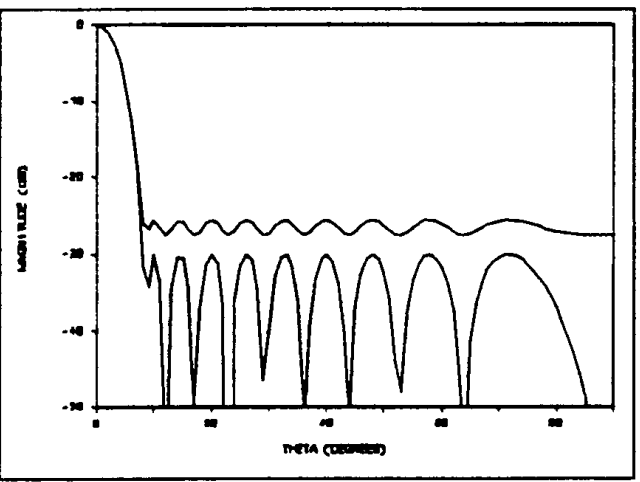

Figure 15. Expected and design radiation patterns (x'z'-plane) for a 20 element, DolphChebyshev synthesized linear array with $\lambda / 2$ spacing and a $-30 \mathrm{~dB}$ design side lobe level

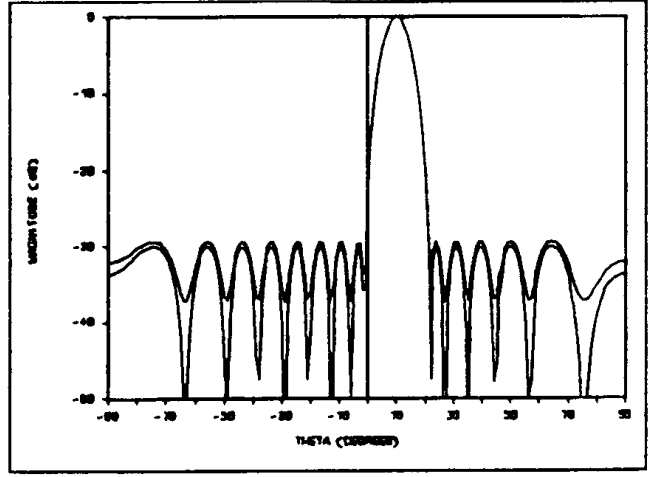

Figure 16. Expected and design radiation patterns ( $x^{\prime} z^{\prime}$-plane) for a $15 \times 15$ element, Dolph-Chebyshev synthesized array with $\lambda / 2$ spacing and a $-30 \mathrm{~dB}$ design side lobe level scanned at $10^{\circ}$ from broadside

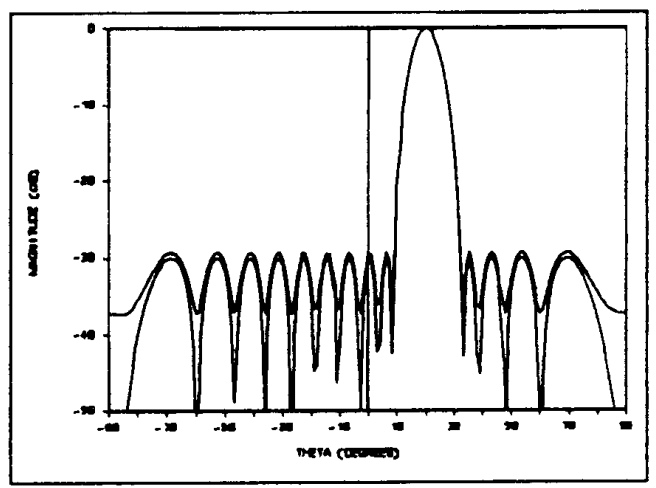

Figure 17. Expected and design radiation patterns ( $x^{\prime} z^{\prime}$-plane) for a $15 \times 15$ element. Dolph-Chebyshev synthesized array with $\lambda 2$ spacing and a $-30 \mathrm{~dB}$ design side lobe level scanned at $20^{\circ}$ from broadside 


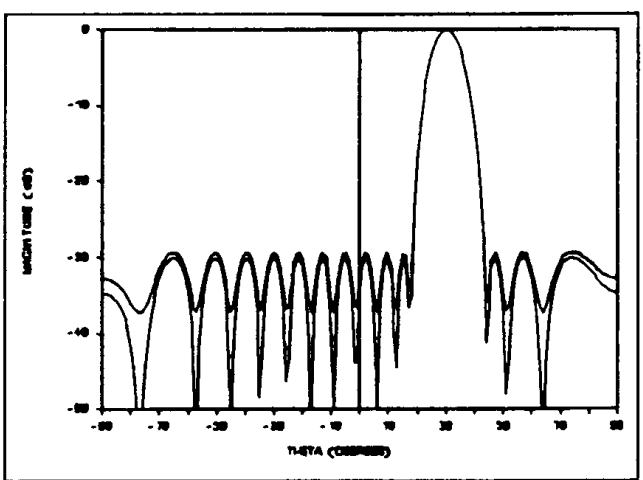

Figure 18. Expected and design radiation patterns ( $x^{\prime} z^{\prime}$-plane) for a $15 \times 15$ element, Dolph-Chebyshev synthesized array with $\lambda / 2$ spacing and a $-30 \mathrm{~dB}$ design side lobe level scanned at $30^{\circ}$ from broadside

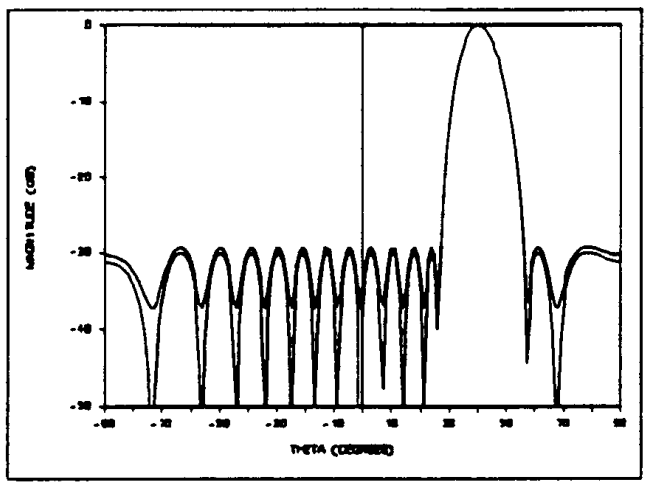

Figure 19. Expected and design radiation patterns ( $x^{\prime} z^{\prime}$-plane) for a $15 \times 15$ element. Dolph-Chebyshev synthesized array with $\lambda / 2$ spacing and $\mathrm{a}-30 \mathrm{~dB}$ design side lobe level scanned at $40^{\circ}$ from broadside

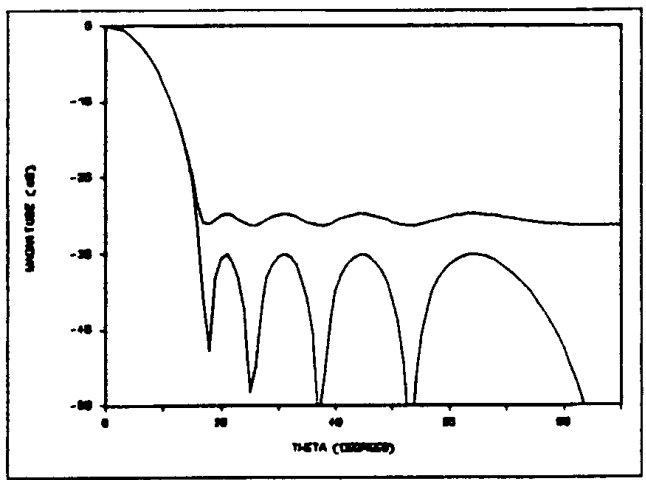

Figure 20. Expected and design radiation pattems ( $x^{\prime} z^{\prime}$-plane) for a 10X10 element. Dolph-Chebyshev synthesized array with $\lambda / 2$ spacing and a $-30 \mathrm{~dB}$ design side lobe level (ail errors present, Trend 5)

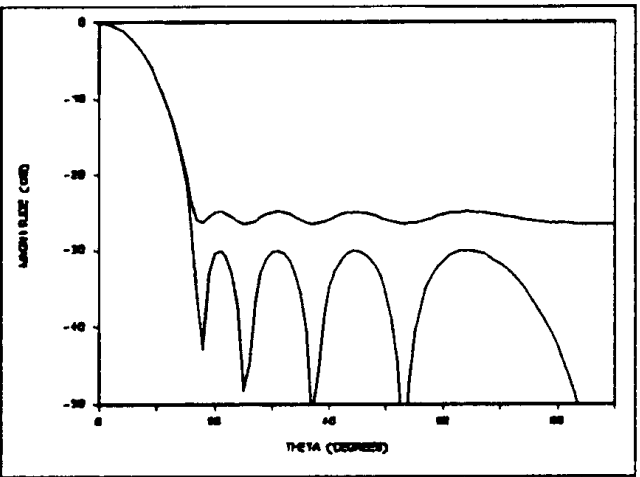

Figure 21. Expected and design radiation patterns ( $x^{\prime} z^{\prime}$-plane) for a $10 \times 10$ element. Dolph-Chebyshev synthesized array with $\lambda / 2$ spacing and $\mathrm{a}-30 \mathrm{~dB}$ design side lobe level (phase error set to 0, Trend 5) 


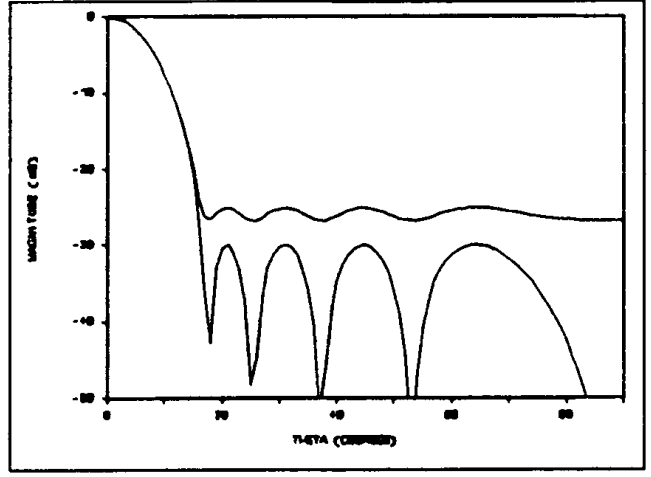

Figure 22. Expected and design radiation patterns ( $x^{\prime} z^{\prime}$-plane) for a $10 \times 10$ element, Dolph-Chebyshev synthesized array with $\lambda / 2$ spacing and a $-30 \mathrm{~dB}$ design side lobe level (phase and amplitude errors set to 0 , Trend 5

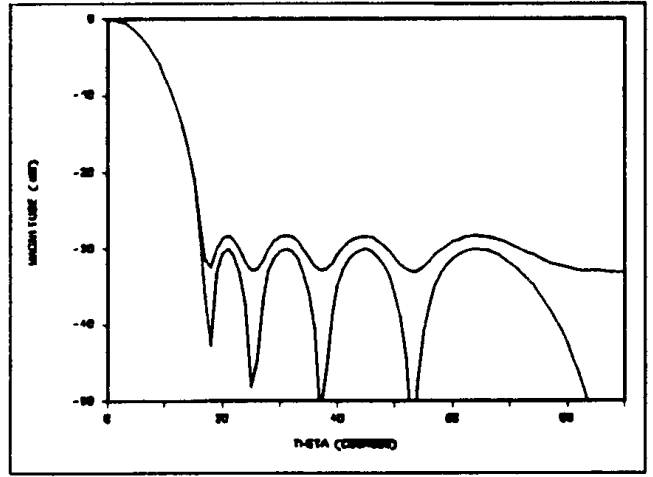

Figure 23. Expected and design radiation patterns ( $x^{\prime} z^{\prime}$-plane) for a $10 \times 10$ element, Dolph-Chebyshev synthesized array with $\lambda / 2$ spacing and a $-30 \mathrm{~dB}$ design side lobe level (amplitude and phase errors set to $0 ; P=1$, Trend 5)

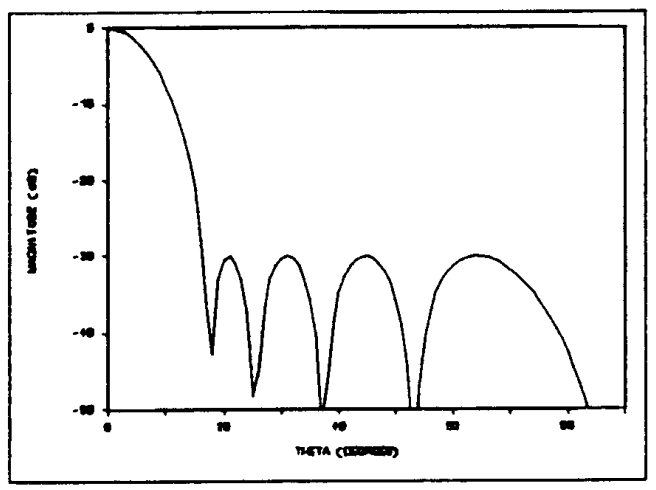

Figure 24. The expected (no-errors) and design radiation patterns ( $x^{\prime} z^{\prime}$-plane) for a $10 \times 10$ element. Dolph-Chebyshev synthesized array with $\lambda / 2$ spacing and a $-30 \mathrm{~dB}$ design side lobe level

\section{BIBLIOGRAPHY}

1. Allen. J.L et al. Phased Array Radar Studies: Technical Report No. 236. 1 July 1960 1 July 1961. Lexington MA: Massachusetts Institute of Technology, Lincoln Laboratory, 13 November 1961 (AD-271724).

2. Ashmead. D. "Optimum Design of Linear Arrays in the Presence of Random Errors," 1.R.E. Transactions on Antennas and Propagation, AP-4: 81-92 (December 1952).

3. Bailin. L.L. and M.J. Ehrlich. "Factors Affecting the Performance of Linear Arrays," Proceeding of the I.R.E.41: 235-241 (February 1953).

4. Balanis, Constatine A. Antenna Theory Analysis and Design. New York: Harper \& Row, Publishers, 1982.

5. Bresier, A.D. "A New Algorithm for Calculating the Current Distributions of Dolph-Chebyshev Arrays," I.E.E.E. Transactions on Antennas and Propagation, AP-28: 951-952 (November 1980). 
6. Burns, M.A., S.R. Laxpati, and J.P. Shelton, Jr. "A Comparitive Sludy of Lnear Array Synthesis Technique Using a Personal Computer," I.E.E.E. Transactions on Antennas and Propagation, AP-32: 884-887 (August 1987).

7. Cheng, D.K. "Effect of Arbitrary Phase Errors on the Gain and Beamwidth Characteristics of Radiation Pattem," I.R.E. Transactions on Antennas and Propagation AP-3: 145-147 (July 1955).

8. Chrisman. Bradley P. "Planar Array Antenna Design Analysis," Master's Thesis, School of Engineering. Air Force Institute of Technology, Air University, 1989, Document Number AFIT GE ENG 89D-4.

9. Dwight. H.B. Tables of Integrals and Other Mathematical Data. New York: The Macmillan Company, 1947.

10. Elliott. Robert S. Antenna Theory and Design. Englewood Cliffs $\mathrm{NJ}$ : Prentice-Hall, Inc., 1981.

11. Elliott. Robert S. "Mechanical and Electrical Tolerances for Two Dimensional Scanning Antenna Arrays," I.R.E. Transactions on Antennas and Propagation, AP-6: 114-120 (January 1958).

12. Elliott. Robert S. "On Discretizing Continuous Aperture Distributions," I.E.E.E. Transactions on Antennas and Propagation, $A \bar{P}-25$ : 617-621 (September 1977).

13. Elliott, Robert S. "The Theory of Antenna Arrays." Microwave Scanning Antennas, Volume 2. edited by R.C. Hansen. New York: Academic Press, 1966.

14. Gilbert, E.N. and S.P. Morgan. "Optimum Design of Directive Antenna Arrays Subject to Random Variations," Bell Systems Technical Journal, 34: 637-663 (May 1955).

15. Goldstein. H. Classical Mechanics. Reading, MA: Addison-Wesly, 1950.
16. Leichter, M. "Beam Pointing Errors of Long Line Sources," I.R.E. Transactions on Antennas and Propagation, AP-8: 268-275 (May 1960).

17. Parsons, Robert. Statistical Analysis: $\underline{A}$ Decision-Making Approach (Second Edition). Now York: Harper and Row, Publishers, 1978.

18. O'Neill, H.F. and Bailin, L.L. "Further Effects of Manufacturing Tolerances on the Performance of Linear Shunt Arrays," I.R.E. Transactions on Antennas and Propagation, AP-4: 93-102 (December 1952).

19. Pozar, David M. Antenna Design Using Personal Computers. Dedham MA: Artech House, Inc., 1985.

20. Rondinelli. L.A. "Effects of Random Errors on the Performance of Antenna Arrays of Many Elements," I.R.E. National Convention Record, Pant 1: 174-189 (1959)

21. Ruze, J. "The Effect of Aperture Errors on the Antenna Radiation Pattern ," Nuovo Cimento, 9 Supplement 3: 364-380 (1952).

22. Skolnik, Merrill I. "Nonuniform Arrays." Antenna Theory, Part 1, edited by R.E. Collin and F.J. Zucker. New York: McGraw Hill Book Company, 1969.

23. Stutzman. Warren L. and Gary A. Thiele. Antenna Theory and Design. New York: John Wiley \& Sons, 1981 .

24. The Ballistic Missile Defense Advanced Technology Center, U.S. Army. Contract DASG60-77-C-0078 with Department of Industrial and Management Systems Engineering, Pennsylvania State University. Huntsville AL. 11 June 1979. 